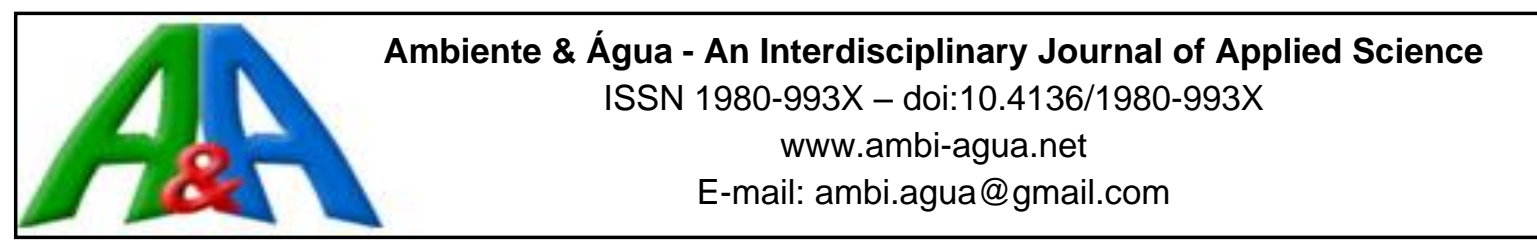

\title{
Metals and dissolved organic carbon (DOC) of surface waters in two adjacent watersheds in the eastern Amazon
}

\author{
ARTICLES doi:10.4136/ambi-agua.2377
}

Received: 11 Feb. 2019; Accepted: 01 Oct. 2019

\section{Juliana Feitosa Felizzola ${ }^{*}$; ; Anthony D. Cak ${ }^{2} \mathbb{D}^{-}$; Ricardo de Oliveira Figueiredo ${ }^{3}$; Marcelo de Oliveira Lima ${ }^{4}$ in}

${ }^{1}$ Laboratório de Análises de Sistemas Sustentáveis (LASS). Embrapa Amazônia Oriental (EMBRAPA), Travessa Dr. Enéas Pinheiro, s/n, Caixa Postal 48, CEP: 66.095-100, Belém, PA, Brazil ${ }^{2}$ Environmental Sciences Initiative, Advanced Science Research Center at The Graduate Center.

The City University Of New York (CUNY), 85St, CEP: 10031, NY, United States. E-mail: acak@ gc.cuny.edu ${ }^{3}$ Embrapa Meio Ambiente, Rodovia SP-340, Km 127,5, CEP: 13918-110, Jaguariúna, SP, Brazil. E-mail: ricardo.figueiredo@embrapa.br

${ }^{4}$ Instituto Evandro Chagas (IEC). Seção Meio Ambiente. Embrapa Meio Ambiente (EMBRAPA), Rodovia BR316, km 7, s/n, CEP: 67030-000, Ananindeua, PA, Brazil. E-mail: marcelolima@iec.gov.br *Corresponding author. E-mail: juliana.felizzola@embrapa.br

\begin{abstract}
Land-use intensification in the "Zona Bragantina" region, an area in the eastern Brazilian Amazon, has accelerated landscape changes from primary forest to a mixture of different land uses: native forest (riparian vegetation), secondary forest, pasture, annual cropland, perennial cropland, bare soil and fallow vegetation, with negative consequences for the stability and equilibrium of river systems. This study examined the temporal and spatial variation of dissolved organic carbon (DOC) and metal concentrations in stream water of the Cumaru (ICU) and São João (ISJ) watersheds from May 2014 to April 2015, to understand the chemical changes in forests and agro-ecosystems during one year. In both watersheds, higher concentrations of dissolved metals (aluminum, sodium, barium, calcium, iron, potassium, magnesium, copper and zinc) and DOC were measured in the rainy season, with considerable variability due to precipitation events. Furthermore, strong spatial variation was observed, with lower conductivity, higher $\mathrm{pH}$, and higher concentrations of DOC and dissolved metals in the downstream reaches, especially for DOC in a small reservoir below a dam in ISJ. Significant human intervention was located in these downstream areas, including nearby agriculture and pasture, and use of stream resources for domestic activity. Sandy soil in these areas likely facilitated leachate processes, mainly in deforested areas, supporting the increased movement of metals from the land to the stream.
\end{abstract}

Keywords: amazon basin, stream, water quality.

\section{Metais e carbono orgânico dissolvido (COD) de águas superficiais em duas bacias adjacentes na Amazônia Oriental}

\section{RESUMO}

A intensificação do uso da terra na região da "Zona Bragantina", uma área da Amazônia Oriental brasileira, acelerou as mudanças de paisagem da floresta primária para uma mistura de 
diferentes usos da terra: floresta (vegetação ripária), floresta secundária, pastagem, terra agrícola anual, terra cultivada perene, solo descoberto e vegetação em pousio, com consequências negativas na estabilidade e equilíbrio dos sistemas fluviais. Este estudo examinou as variações temporais e espaciais das concentrações de carbono orgânico dissolvido (COD) e de metais em água fluvial das bacias hidrográficas Cumaru (ICU) e São João (ISJ), de maio de 2014 a abril de 2015 para entender as variações químicas nos agrossistemas e florestas durante 1 ano de monitoramento. Em ambas as bacias, maiores concentrações de metais dissolvidos (alumínio, sódio, bário, cálcio, ferro, potássio, magnésio, cobre e zinco) e COD foram medidos na estação chuvosa, com considerável variabilidade devido a eventos de precipitação. Além disso, foi observada forte variação espacial, com baixa condutividade, $\mathrm{pH}$ mais alto e maiores concentrações de COD e metais dissolvidos nos trechos a jusante, especialmente para COD em um pequeno reservatório abaixo de uma barragem no ISJ. Estas áreas a jusante sofrem intervenção humana significativa, incluindo agricultura e pastagem nas proximidades e uso dos recursos hídricos para a atividade doméstica. O solo arenoso nestas áreas provavelmente facilita os processos de lixiviação, principalmente em áreas desmatadas, apoiando o aumento do movimento de metais da paisagem para o córrego.

Palavras-chave: bacia amazônica, córrego, qualidade da água.

\section{INTRODUCTION}

Together with climate, the amount and composition of trace metals and other biogeochemical elements in stream water have been shown to be a function of several landscape-level factors. These include the underlying geochemistry and geomorphology (e.g., weathering), as well as land-cover change (LC) and other anthropogenic influences occurring within watersheds (Das et al., 2009). Understanding the composition and fate of metals is critical, since they do not degrade but are instead mobilized and remobilized through the ecosystem. Furthermore, many of these metals can present serious public and environmental health effects, including toxicity at high concentrations. While widely studied in temperate systems around the world, the fate of metals in the tropics has received less attention (Janeau et al., 2014). Metals in small watersheds of the Amazon Basin in particular have been scarce, with only one study limited to analysis of trace metals in large rivers.

In aquatic systems, understanding metallic transport and its bioavailability requires knowledge of the processes that are linked with parameters such as dissolved organic carbon (DOC), suspended particulate matter, other organic and inorganic binders, colloids, as well as $\mathrm{pH}$ and electrical conductivity specific to the water (Huser et al., 2001). The interaction of DOC with dissolved metals is also influenced by the specific conductance of water, since DOC tends to flocculate at high ionic strength and to be more mobile at low ionic strength (Stumm and Morgan, 1996). According to the literature, the dissolved fraction of a metal in water is not only comprised of "free" elements, but also of complex metal ions with inorganic and organic binders competing with particles for metals with reactions of sorption/complexation (Shafer et al., 1997). Metals can occur in suspended forms as colloids, affecting the reactivity and partitioning of the metal, where the dissolved fractions and metallic particles do not behave independently, due to the dynamic exchange through adsorption-desorption reactions. These reactions are involved in the synthesis of DOC in terrestrial ecosystems by forming strong bonds and complexes with organic matter $(\mathrm{OM})$ and its subsequent transport to aquatic ecosystems through precipitation events, temperature, land cover, etc., buffering of nutrients and pollutants, organics and metal(loid)s, and influencing the global carbon cycle (Bolan et al., 2014).

DOC is a heterogeneous mixture of natural organic compounds that is influenced by seasonality and location, due to different organic matter sources and diagenesis (Shafer et al., 1997). The concentration of dissolved metals, as affected by DOC, is thus likely to be 
influenced by seasonal changes like discharge and precipitation, since episodic but intense rain events in the tropics can input allochthonous organic matter which is comprised of humic and fulvic compounds that can complex with metals (Aitkenhead-Peterson et al., 2003).

LC also affects dissolved metal concentrations in stream water, through processes such as agriculture, urban development, and mining (Duann et al., 2017). In some parts of the Amazon Basin, tropical forest has been replaced initially by small-scale subsistence agriculture, followed by large-scale clearing for pasture and other types of agriculture (Wickel et al., 2004). This has created a dynamic mixture of different types of land cover, with important consequences for hydrological and biogeochemical processes, especially for small streams (Cak et al., 2016). The spatial location of land cover within watersheds, coupled with geomorphology, is likely to play an important role in dissolved chemical concentrations and their behavior moving through the aquatic system, such as from soils to stream water and within streams (Wickel et al., 2004). Understanding these processes in small Amazonian streams is important to serve as a complement to data from other studies (Duann et al., 2017).

The goal of this study, then, was to identify the behavior of dissolved metals and DOC concentrations in small streams in the Amazon Basin during one year of monitoring to determination background levels due the potential toxicity to biota. We hypothesized that land cover is likely to play an important role in DOC and metal interactions as a result of anthropogenic impacts related to agricultural activities. This effect is also likely driven by seasonal variability in relation to how climate changes.

\section{MATERIALS AND METHODS}

\subsection{Description of the study area}

The study area comprised two small watersheds, Cumaru (4,121.25 ha) (ICU) and São João (2,644.79 ha) (ISJ), in the municipality of Igarapé-Açu, Pará, Brazil (Figure 1).

These areas were chosen because the watersheds are part of a large network of streams within the Maracanã River Basin, located in the Bragantina region of northeastern Pará. The drainage systems of this area are classified as perennial watercourses. The drainages are from 1st and 2nd order to the Maracanã River which is hierarchically 3rd order. The main water courses that bathe the municipality of Igarapé-Açu are the Caripi Rivers, Maracanã River, and Jambuaçú Stream which should be a river by its flow. The Cumaru and São João Streams are tributaries of the Maracanã River. Land cover change in this area began in 1883 with the construction of the Bragança Railway. Slash-and-burn agriculture with crop rotation remains the predominant type of land cover, particularly on farms that are approximately 25 ha in size (Kato et al., 2004). More recently, however, cattle ranches and palm oil plantations have expanded into the area (Figure 1).

Prior to field work, the spatial watershed boundaries of ICU and ISJ were delineated using image interpretation from overlay of RapidEye images from 2012 with a spatial dataset of stream locations digitized from a 1:77,000-scale topographic map. These 2012 RapidEye images also were used to identify land use and land cover in both watersheds. This imagery was the most cloud-free imagery set available for our sampling period (2014), although we acknowledge that land use changes likely occurred and/or intensified during this two-year difference. Land cover (LC) was grouped into the following classes: native forest (riparian vegetation), secondary forest, pasture, annual cropland, perennial cropland, bare soil, and fallow vegetation (Figure 1 and Table 1). All land-use and land-cover analyses were conducted by the remote-sensing laboratory of Embrapa Eastern Amazon, in Belém, Brazil.

\subsection{Land cover characteristics}

Land cover varied by sampling location and surrounding area of the sampling site. The headwater reaches were predominantly composed of riparian vegetation and secondary forest, 
while the middle reaches were predominantly secondary forest, perennial agriculture, and pasture. In the lower reaches, land cover was predominantly perennial agriculture, and pasture, with the area surrounding the dam in ISJ containing both pasture and secondary forest. Overall, primary forest made up approximately $10-14 \%$ of each watershed, while secondary forest was approximately $30-50 \%$ of each watershed (Table 1 ).

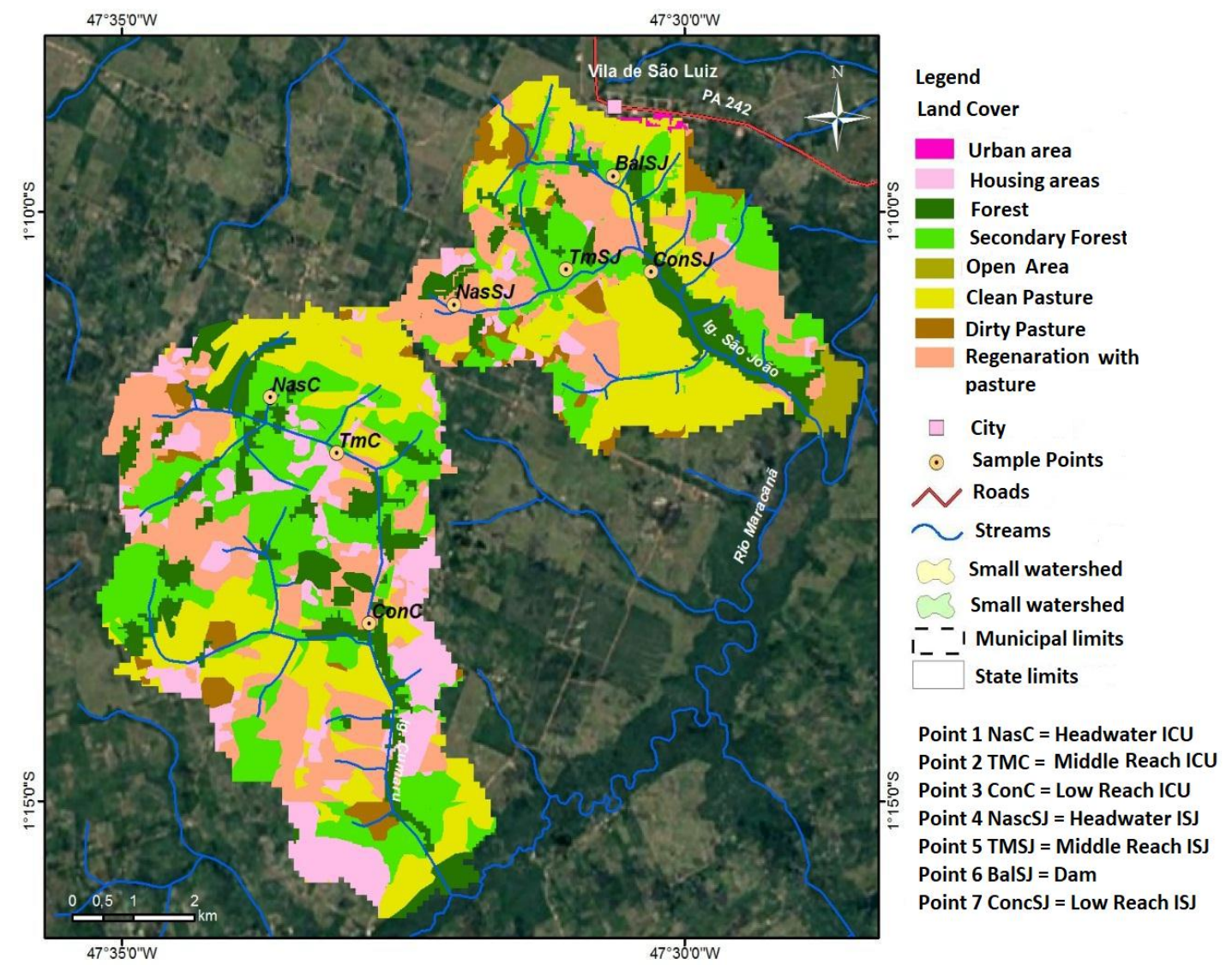

Source: Embrapa Amazônia Oriental 2014

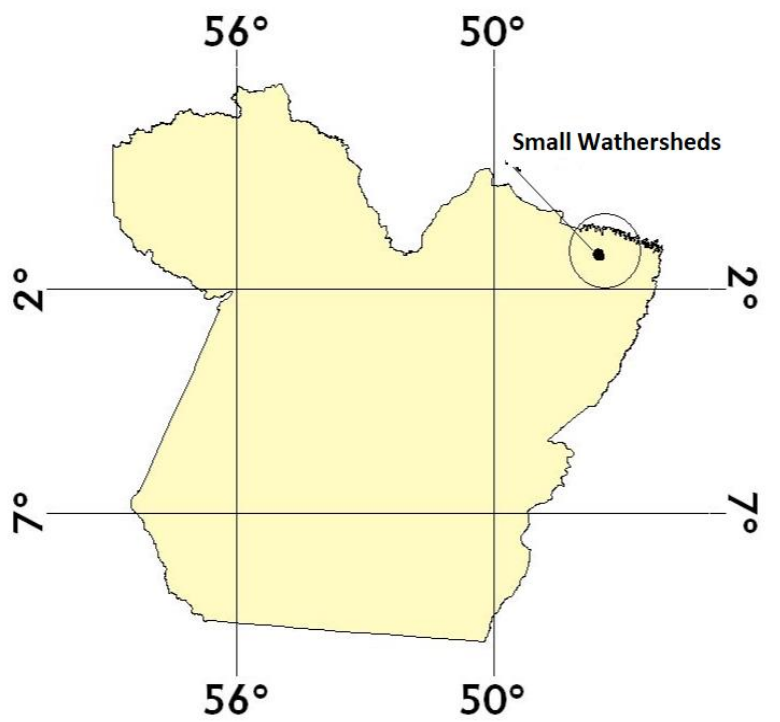

Figure 1. Map showing the location of the two study watersheds. Land cover in the headwater reaches is predominantly riparian vegetation and secondary forest; in the middle reaches, it is predominantly secondary forest, perennial agriculture and pasture; in the lower reaches, it is characterized as perennial agriculture, pasture and secondary forest. 
Table 1. Amount and percent of the total basin of different land cover types in the two study watersheds (Cumaru, ICU; São João, ISJ), as identified from classification of a RapidEye image collected in 2012. A small portion of the image contained cloud cover that masked part of the watershed land cover, and is noted here in order to complete the percentage calculations.

\begin{tabular}{lccccc}
\hline \multirow{2}{*}{ Land cover } & \multirow{2}{*}{ Sample Points } & \multicolumn{2}{c}{ ICU } & \multicolumn{2}{c}{ ISJ } \\
\cline { 3 - 6 } & & Area (ha) & $\%$ & Area (ha) & $\%$ \\
\hline Forest & 1,4 & 425.03 & 10.31 & 377.40 & 14.27 \\
Secondary Forest & 1,4 & $2,022.60$ & 49.08 & 955.40 & 36.12 \\
Forest + Secondary Forest & 1,4 & 2447.63 & 59.39 & 1332.8 & 50.39 \\
Fallow Vegetation* & $2,3,5,6$ & - & - & 80.43 & 3.04 \\
Pasture & 3,7 & $1,014.40$ & 24.61 & 916.53 & 34.65 \\
Annual Crops & $2,3,5,6$ & 248.29 & 6.02 & 81.37 & 3.08 \\
Perennial Crops & $2,3,5,6$ & 284.14 & 6.89 & 120.37 & 4.55 \\
Bare Soil & 3,7 & 6.90 & 0.17 & 27.81 & 1.05 \\
Water & - & 5.00 & 0.12 & 0.48 & 0.02 \\
Clouds & - & 114.50 & 2.79 & 85.01 & 3.21 \\
Total Basin Area & - & $4,121.25$ & - & $2,644.79$ & - \\
\hline
\end{tabular}

*Fallow Vegetation: refers to the practice of leaving fields unplowed in rotation - when a field lies fallow, the soil regains nutrients that are sucked up by over-planting.

Agriculture in this region is typically found on small-holding farms of less than 50 ha in size, with traditional crops such as cassava, corn, beans, rice, pepper, and passion fruit. The amount of pasture and perennial agriculture in the studied watersheds differed, comprising approximately $25 \%$ of the area in ICU and $7 \%$ in ISJ. In contrast, the amount of forest area was similar between both watersheds, likely due to riparian wetlands found in ISJ.

Geomorphological characteristics of ICU and ISJ are representative of the region, which was formed by ancient alluvial sediments belonging to the Tertiary Barreiras series and the Pleistocene. Sediments in this area also include more recent alluvial deposits containing a small saline influence (Lima et al., 2007). Consequently, the landscape relief of the area is characterized by long undulations. Soils in the area are diverse and influenced by ancient alluvial terraces, and are characterized as a mixture of Dystrophic Ultisols with sandy texture, medium/clayey Dystrophic Ultisols, medium/clayey concretionary Dystrophic Ultisols, and medium texture Dystrophic Yellow Oxisols. Such soils are deep, well-drained, and permeable, with good moisture retention capacity. While they are acidic and nutrient-poor, these soils have good potential for tropical agriculture through the use of lime and fertilizer to increase their productivity.

Climate in the region is Am on the Köppen-Geiger scale, hot and humid with annual rainfall around 2,500 $\mathrm{mm}$. The dry season usually occurs from September to December and the rainy season occurs between March and June. Precipitation events are divided into four periods: rainy season (March and April 2014 for some variables, May and June 2014, and March and April 2015 for some variables), transition season from rainy to dry (T1, July and August 2014), dry season (September 2014, October 2014, November 2014, and December 2014) and transition season from dry to rainy (T2, January and February 2015). The transition seasons, rainy to dry and dry to rainy, occur from July to August and January to February, respectively. Monthly average temperatures range between 26 and $28^{\circ} \mathrm{C}$, while the yearly average relative 
humidity is $85 \%$. During this study, daily meteorological data (e.g., precipitation and air temperature) were collected at an Embrapa Eastern Amazon weather station located in the Cumaru watershed (the São João watershed is located nearby, so data were aggregated for both watersheds).

\subsection{Sampling and chemical analysis}

In both ICU and ISJ, seven sampling stations were established - three in ICU (corresponding to the headwater, middle reach, and lower reach) and four in ISJ (corresponding to the headwater, middle reach, dam, and lower reach). In ISJ, a small dam was constructed by the community to allow a road to pass over the stream; it is a location of frequent domestic activities (e.g., laundry, bathing, subsistence fishing). Two sets of water samples were collected at each sampling location monthly between March 2014 and April 2015, for a total of seven sampling sites and 98 observations. Water samples were collected at 20-25 cm below the surface and duplicate samples for trace metal analysis were collected in $250 \mathrm{~mL}$ polypropylene bottles (wide mouth $\varnothing=4 \mathrm{~cm}$ ) (APHA et al., 1998). Triplicate samples for dissolved organic carbon (DOC) analysis were collected in borosilicate bottles. At the time of sample collection, other physiochemical variables ( $\mathrm{pH}$, electrical conductivity (EC) and temperature) were measured in the field with a multiparameter probe (Horiba Model HI 9828). Water flow was measured following the method of Global Water Instrumentation (2004).

All water samples were analyzed at a laboratory of the Embrapa Eastern Amazon and at the Evandro Chagas Institute in Belém, Brazil. Duplicate samples for trace metals and other water chemistry variables were filtered through $0.45 \mu \mathrm{m}$ acetate cellulose membranes, acidified with ultrapure $\mathrm{HNO}_{3}$ to $\mathrm{pH}<2$, and stored at $4^{\circ} \mathrm{C}$ until analysis using an optical emission spectrometer with inductively coupled plasma analyzer (VISTA-MPX, Varian Inc.). These included samples for aluminum (Al), barium (Ba), calcium $(\mathrm{Ca})$, cadmium $(\mathrm{Cd})$, cobalt $(\mathrm{Co})$, chromium $(\mathrm{Cr})$, copper $(\mathrm{Cu})$, iron $(\mathrm{Fe})$, potassium $(\mathrm{K})$, magnesium $(\mathrm{Mg})$, manganese $(\mathrm{Mn})$, sodium $(\mathrm{Na})$, nickel $(\mathrm{Ni})$, lead $(\mathrm{Pb})$, and zinc $(\mathrm{Zn})$. Triplicate samples for DOC were filtered through Millipore membranes glass fiber filters with porosity of $0.7 \mu \mathrm{m}$ which are muffled at $450^{\circ} \mathrm{C}$, and then stored in (calcined) quartz Millipore $(40 \mathrm{~mL})$ bottles, kept with $1 \mathrm{ml}$ of phosphoric acid $\left(10 \% \mathrm{H}_{3} \mathrm{PO}_{4}\right)$ and refrigerated until carbon analysis (Shimadzu TOC-V Model CSN).

Monthly hydro biogeochemical mass fluxes were estimated for each sampling site using Equation 1.

$$
F m=\frac{C \times Q \times 86.400 \times T}{10^{6}}
$$

Where Fm is element mass flux in $\mathrm{kg}_{\text {month }}{ }^{-1}$; $\mathrm{C}$ is element concentration in $\mathrm{mg} \mathrm{L}^{-1}$; $\mathrm{Q}$ is discharge in $\mathrm{L} \mathrm{s}^{-1}$; $\mathrm{T}$ is time in days; 86.400 is the factor to convert seconds to days; and, $10^{6}$ is the factor to convert $\mathrm{mg}$ month $^{-1}$ to $\mathrm{kg}$ month $^{-1}$. Hydrochemical flow calculations of each studied watershed were performed from mean of the dissolved ion concentration and flow-rate data obtained (Table 2: Mean All data 2014-2015; Metal in $\mu \mathrm{g} . \mathrm{mL}^{-1}$ ). Flow rate and concentration were considered constant for each campaign from the 15-day period leading up to the following campaign, totaling about 30 days of the same concentration for the purposes of this estimate.

The month mass flow in $\mathrm{kg} \cdot \mathrm{ha}^{-1}$ was obtained by adding the monthly hydrochemical flows and divided by the total area of the watershed (4,121.25 ha for ICU and 2,644.79 ha for ISJ) (Barroso, 2011). 
Table 2. Summary of means and intra- and inter- annual variability: Cumaru and São João.

\begin{tabular}{|c|c|c|c|c|c|c|}
\hline Filterable (ug. $\mathrm{L}^{-1}$ ) & ICU & & & & & \\
\hline \multirow[t]{2}{*}{ Parameter } & Metal & Mean Rainy Season (rsd) & Mean Transitional Season (rsd) & Mean Dry Season (rsd) & Mean Transitional Season (rsd) & Mean (rsd) \\
\hline & & $\begin{array}{l}\text { March, April, May, June } \\
\text { n= } 4\end{array}$ & $\begin{array}{l}\text { July, August } \\
\quad \mathbf{n}=2\end{array}$ & $\begin{array}{c}\text { September, October, November, December } \\
n=4\end{array}$ & $\begin{array}{l}\text { January, February } \\
\quad \mathrm{n}=2\end{array}$ & $\begin{array}{l}\text { All data 2014-2015 } \\
\quad \mathrm{n}=12\end{array}$ \\
\hline \multirow[t]{17}{*}{ Headwater } & $\mathrm{Al}$ & $104.76(0.04)$ & $120.52(0.021)$ & $108.80(0.02)$ & $68.51(0.06)$ & $102.70(0.04)$ \\
\hline & $\mathrm{Ba}$ & $31.75(0.05)$ & $73.59(0.073)$ & $2.12(0.002)$ & $0.82(0.001)$ & $23.69(0.05)$ \\
\hline & $\mathrm{Ca}$ & $278.09(0.16)$ & $376.94(0.23)$ & $166.50(0.20)$ & $98.34(0.05)$ & $227(0.20)$ \\
\hline & $\mathrm{Cd}$ & $0.40(13 * 10-5)$ & $0.2185(8.15 * 10-5)$ & $0.30(0)$ & $0.31(1 * 10-5)$ & $0.34(8 * 10-5)$ \\
\hline & Co & $0.67(0.0002)$ & $0.50(0)$ & $0.99(0.0006)$ & $2.93(0.0024)$ & $1.12(0.0013)$ \\
\hline & $\mathrm{Cr}$ & $0.40(0)$ & $0.816(6.45 * 10-5)$ & $0.40(0)$ & $1.35(0.00095)$ & $0.63(0.00053)$ \\
\hline & $\mathrm{Cu}$ & $5.17(0.004)$ & $10.83(6.23 * 10-3)$ & $7.13(0.0048)$ & $8.21(0.0010)$ & $7.27(0.0045)$ \\
\hline & $\mathrm{Fe}$ & $57.70(0.09)$ & $14.26(0.008)$ & $29.02(0.04)$ & $11.70(0.01)$ & $33.23(0.06)$ \\
\hline & $\mathrm{K}$ & $51.84(0.03)$ & $18.37(0.011)$ & $4.37(0.0034)$ & $54.02(0.050)$ & $30.80(0.04)$ \\
\hline & $\mathrm{Mg}$ & $246.80(0.04)$ & $0.22(0.010)$ & $241(0.03)$ & $229.24(0.01)$ & $237.42(0.033)$ \\
\hline & $\mathrm{Mn}$ & $1.78(0)$ & $1.60(1 * 10-4)$ & $1.06(0.0006)$ & $2.22(0.0010)$ & $1.58(0.001)$ \\
\hline & $\mathrm{Na}$ & $1758.71(0.68)$ & $1768.91(0.053)$ & $1412.78(0.08)$ & $1185.91(0.27)$ & $1549.63(0.52)$ \\
\hline & $\mathrm{Ni}$ & $3.99(0.002)$ & $1.50(0)$ & $2.97(1.13)$ & $3.08(3 * 10-4)$ & $3.08(0.002)$ \\
\hline & $\mathrm{Pb}$ & $5(0.00)$ & $5(0.00)$ & $5.83(0.002)$ & $6.19(0.001)$ & $5.48(0.001)$ \\
\hline & $\mathrm{Zn}$ & $30.16(0.04)^{1}$ & $49.93(0.044)$ & $1.01(0.001)$ & $6.64(0.005)$ & $18.88(0.03)$ \\
\hline & Discharge $\left(\right.$ L.s $\left.^{-1}{ }^{1}\right)$ & $4.60(2.33)^{2}$ & $3.05(1.42)$ & $1.77(0.32)$ & $1.44(0.04)$ & $2.87(1.98)^{2}$ \\
\hline & TOC $\left(m g . L-{ }^{-1}\right) n=5$ & $0.71(0.18)$ & $1.47(0.96)$ & $0.76(0.29)$ & $0.62(0.06)$ & $0.83(0.51)^{2}$ \\
\hline \multirow[t]{17}{*}{ Middle Reach } & $\mathrm{Al}$ & $148.7(0.07)$ & $67.13(0.017)$ & $52.35(0.01)$ & $45.91(0.05)$ & $85.96(0.06)$ \\
\hline & $\mathrm{Ba}$ & $38.65(0.06)$ & $64.34(0.063)$ & $1.74(0.001)$ & $3.13(0.003)$ & $24.71(0.05)$ \\
\hline & $\mathrm{Ca}$ & $660.37(0.39)$ & $635.41(0.06)$ & $692.69(0.11)$ & $920.85(0.08)$ & $710(0.25)$ \\
\hline & $\mathrm{Cd}$ & $0.32(4 * 10-5)$ & $0.3(0)$ & $0.32(0.00003)$ & $0.43(1.3 * 10-4)$ & $0.33(7 * 10-5)$ \\
\hline & Co & $0.68(0.0003)$ & $0.57(3.04 * 10-4)$ & $0.77(0.0004)$ & $2.03(0.0015)$ & $0.92(0.00086)$ \\
\hline & $\mathrm{Cr}$ & $0.40(0)$ & $0.68(1.8 * 10-4)$ & $0.41(2 * 10-5)$ & $1.53(0.00079)$ & $0.64(0.000528)$ \\
\hline & $\mathrm{Cu}$ & $5.43(0.01)$ & $1.37(7.65 * 10-4)$ & $1.06(0.0005)$ & $1.75(0.0010)$ & $2.68(0.00511)$ \\
\hline & $\mathrm{Fe}$ & $240.95(0.13)$ & $167.85(0.20)$ & $183.19(0.02)$ & $232.21(0.04)$ & $208.06(0.09)$ \\
\hline & $\mathrm{K}$ & $99.26(0.08)$ & $25.32(0.009)$ & $11.07(0.0010)$ & $124.99(0.113)$ & $61.83(0.08)$ \\
\hline & $\mathrm{Mg}$ & $328.15(0.09)$ & $0.33(0.020)$ & $384.76(0.37)$ & $417.58(0.03)$ & $362.30(0.065)$ \\
\hline & $\mathrm{Mn}$ & $5.39(0)$ & $5.05(5 * 10-5)$ & $3.90(0.0011)$ & $6.80(0.0024)$ & $5.07(0.002)$ \\
\hline & $\mathrm{Na}$ & $1932.07(0.68)$ & $1773.77(0.40)$ & $1486.89(0.11)$ & $1438.52(0.36)$ & $1668.37(0.50)$ \\
\hline & $\mathrm{Ni}$ & $3.07(0.001)$ & $1.50(0)$ & $3.03(0.99)$ & $2.37(9 * 10-4)$ & $2.68(0.002)$ \\
\hline & $\mathrm{Pb}$ & $14.02(0.010)$ & $6.19(0.0012)$ & $5(0.00)$ & $6.93(0.002)$ & $8.53(0.007)$ \\
\hline & $\mathrm{Zn}$ & $37.57(0.05)^{1}$ & $36.09(0.035)$ & $0.20(0)$ & $6.09(0.006)$ & $17.99(0.03)$ \\
\hline & Discharge $\left(\right.$ L.s $\left.^{-1}{ }^{1}\right)$ & $142.17(65.63)^{2}$ & $106.78(9.90)$ & $37.96(19.92)$ & $44.40(4.53)$ & $81.78(59.81)$ \\
\hline & TOC (mg.L-1) & $3.61(2.98)$ & $1.56(0.20)$ & $1.40(0.38)$ & $1.53(0.13)$ & $2.29(2.13)^{2}$ \\
\hline
\end{tabular}




\begin{tabular}{|c|c|c|c|c|c|c|}
\hline Continued... & & & & & & \\
\hline \multirow[t]{17}{*}{ Lower Reach } & $\mathrm{Al}$ & $221.13(0.11)$ & $81.33(0.007)$ & $71.38(0.02)$ & $55.48(0.06)$ & $120.30(0.10)$ \\
\hline & $\mathrm{Ba}$ & $45.66(0.07)$ & $60.58(0.060)$ & $1.62(0.001)$ & $2.68(0.0003)$ & $26.30(0.06)$ \\
\hline & $\mathrm{Ca}$ & $837.49(0.23)$ & $486.11(0.53)$ & $127.53(0.09)$ & $913.27(0.10)$ & $554.91(0.40)$ \\
\hline & $\mathrm{Cd}$ & $0.30(0)$ & $0.26(3.88 * 10-5)$ & $0.30(0)$ & $0.70(2.5 * 10-4)$ & $0.36(1.8 * 10-4)$ \\
\hline & Co & $0.75(0.0004)$ & $0.50(0)$ & $0.93(0.0007)$ & $2.82(0.0023)$ & $1.11(0.0013)$ \\
\hline & $\mathrm{Cr}$ & $0.40(0.0004)$ & $0.54(1.4 * 10-4)$ & $10.46(0.0001)$ & $2.46(0.00140)$ & $0.86(0.001)$ \\
\hline & $\mathrm{Cu}$ & $0.75(0.0003)$ & $0.67(7.05 * 10-5)$ & $0.78(0.0002)$ & $0.98(0.0004)$ & $0.79(0.00003)$ \\
\hline & $\mathrm{Fe}$ & $376.45(0.06)$ & $100.20(0.1)$ & $38.97(0.04)$ & $218.62(0.03)$ & $191.61(0.16)$ \\
\hline & $\mathrm{K}$ & $100.34(0.07)$ & $20.13(0.008)$ & $10.77(0.0028)$ & $134.62(0.1222)$ & $62.83(0.08)$ \\
\hline & $\mathrm{Mg}$ & $360.66(0.04)$ & $0.27(0.041)$ & $276.68(0.27)$ & $440.08(0.04)$ & $330.52(0.076)$ \\
\hline & $\mathrm{Mn}$ & $6.91(0)$ & $2.99(0.0020)$ & $1.64(0.0018)$ & $7.51(0.0024)$ & $4.60(0.003)$ \\
\hline & $\mathrm{Na}$ & $1915.08(0.80)$ & $1759.64(0.43)$ & $1469.58(0.09)$ & $1466.03(0.42)$ & $1665.83(0.57)$ \\
\hline & $\mathrm{Ni}$ & $1.71(0)$ & $1.67(1.73 * 10-4)$ & $2.40(0.73)$ & $4.26(0.0027)$ & $2.36(0.002)$ \\
\hline & $\mathrm{Pb}$ & $7.22(0.004)$ & $7.03(0.0020)$ & $5.81(0.001)$ & $5(0.00)$ & $6.35(0.003)$ \\
\hline & $\mathrm{Zn}$ & $46.7(0.07)^{1}$ & $39.21(0.038)$ & $0.20(0)$ & $4.20(0.004)$ & $20.70(0.04)$ \\
\hline & Discharge $\left(\right.$ L.s $\left.{ }^{-1}\right)$ & $249.04(110.77)^{2}$ & $177.62(0.50)$ & 86.18 (41.35) & $68.21(4.64)$ & $149.73(104.21)^{2}$ \\
\hline & TOC $\left(m g . L^{-1}\right)$ & $3.20(2.29)$ & $1.52(0.28)$ & $1.43(0.27)$ & $1.40(0.14)$ & $2.12(1.67)^{2}$ \\
\hline Filterable (ug.L $\mathrm{L}^{-1}$ ) & ISJ & & & & & \\
\hline \multirow[t]{3}{*}{ Parameter } & Metal & Mean Rainy Season (rsd) & Mean Transitional Season (rsd) & Mean Dry Season (rsd) & Mean Transitional Season (rsd) & Mean (rsd) \\
\hline & & $\begin{array}{l}\text { All data 2014-2015 } \\
\qquad n=4\end{array}$ & $\begin{array}{l}\text { All data 2014-2015 } \\
\qquad \mathrm{n}=2\end{array}$ & $\begin{array}{l}\text { All data 2014-2015 } \\
\qquad n=4\end{array}$ & $\begin{array}{l}\text { All data 2014-2015 } \\
\qquad \begin{array}{l}n=2 \\
\text { a }\end{array}\end{array}$ & $\begin{array}{c}\text { All data 2014-2015 } \\
n=12\end{array}$ \\
\hline & & March, April, May, June & July, August & September, October, November, December & January, February & \\
\hline \multirow[t]{17}{*}{ Headwater } & $\mathrm{Al}$ & $104.79(0.07)$ & $126.19(0.038)$ & $49.65(0.01)$ & $66.02(0.03)$ & $83.51(0.05)$ \\
\hline & $\mathrm{Ba}$ & $44.77(0.07)$ & $72.62(0.07)$ & $2.37(0.001)$ & $1.81(0.002)$ & $28.12(0.06)$ \\
\hline & $\mathrm{Ca}$ & $392.41(0.35)$ & $601.42(0.15)$ & $801.77(0.11)$ & $110.84(0.02)$ & $516.77(0.33)$ \\
\hline & $\mathrm{Cd}$ & $0.30(0)$ & $0.358(5.8 * 10-5)$ & $0.30(0)$ & $0.58(2.5 * 10-4)$ & $0.36(1.4 * 10-4)$ \\
\hline & Co & $1.05(0.0007)$ & $0.50(0)$ & $0.66(0.0003)$ & $2.94(0.00024)$ & $1.15(0.0014)$ \\
\hline & $\mathrm{Cr}$ & $0.40(0)$ & $0.72(3.2 * 10-4)$ & $0.50(0.00017)$ & $1.57(0.00077)$ & $0.68(0.00053)$ \\
\hline & $\mathrm{Cu}$ & $102.51(0.18)$ & $3.83(3 * 10-3)$ & $1.35(0.0008)$ & $0.81(0.0002)$ & $35.40(0.1118)$ \\
\hline & $\mathrm{Fe}$ & $159.25(0.27)$ & $260.06(0.253)$ & $177.31(0.01)$ & $10.66(0.01)$ & $157.31(0.20)$ \\
\hline & $\mathrm{K}$ & $85.50(0.05)$ & $18.78(2 * 10-3)$ & $10.90(0.0015)$ & $65.31(0.055)$ & $46.15(0.05)$ \\
\hline & $\mathrm{Mg}$ & $303.75(0.12)$ & $0.28(0.083)$ & $386.86(0.38)$ & $235.39(0)$ & $315.62(0.095)$ \\
\hline & $\mathrm{Mn}$ & $3.39(0)$ & $3.84(0.0022)$ & $3.59(0.0011)$ & $1.94(0.0007)$ & $3.29(0.003)$ \\
\hline & $\mathrm{Na}$ & $1959.41(0.76)$ & $1770.84(0.37)$ & $1523.96(0.10)$ & $1325.84(0.09)$ & $1677.24(0.52)$ \\
\hline & $\mathrm{Ni}$ & $3.07(0.001)$ & $1.50(0)$ & $2.85(0.96)$ & $2.43(0.0002)$ & $2.63(0.002)$ \\
\hline & $\mathrm{Pb}$ & $9.98(0.005)$ & $5(0.00)$ & $5.63(0.001)$ & $6.46(0.001)$ & $7.11(0.004)$ \\
\hline & $\mathrm{Zn}$ & $40.38(0.05)^{1}$ & $38.43(0.036)$ & $0.20(0)$ & $4.95(0.004)$ & $18.97(0.04)$ \\
\hline & Discharge $\left(L . \mathrm{s}^{-1}\right)$ & $3.08(0.63)^{2}$ & $2.87(0.63)$ & $4.04(0.61)$ & $3.53(0.43)$ & $3.44(0.75)^{2}$ \\
\hline & TOC (mg. $\left.\mathrm{L}^{-1}\right)$ & $0.36(0.24)$ & $0.22(0.03)$ & $0.51(0.13)$ & $0.46(0.03)$ & $0.40(0.19)^{2}$ \\
\hline
\end{tabular}




\begin{tabular}{|c|c|c|c|c|c|c|}
\hline Continued... & & & & & & \\
\hline \multirow[t]{17}{*}{ Middle Reach } & $\mathrm{Al}$ & $120.39(0.07)$ & $69.89(0.020)$ & $47.25(0.01)$ & $35.10(0.03)$ & $76.67(0.05)$ \\
\hline & $\mathrm{Ba}$ & $44.08(0.08)$ & $77.37(0.076)$ & $1.45(0)$ & $1.23(0.001)$ & $28.28(0.06)$ \\
\hline & $\mathrm{Ca}$ & $546.25(0.21)$ & $995.39(0.23)$ & $350.99(0.24)$ & $333.39(0.01)$ & $520.54(0.31)$ \\
\hline & $\mathrm{Cd}$ & $0.30(0)$ & $0.26(3.88 * 10-5)$ & $0.30(0)$ & $0.39(9 * 10-5)$ & $0.32(4.6 * 10-5)$ \\
\hline & Co & $0.61(0.0001)$ & $0.50(0)$ & $1.02(0.0007)$ & $3.26(0.0028)$ & $1.17(0.0015)$ \\
\hline & $\mathrm{Cr}$ & $0.40(0)$ & $0.40(0)$ & $0.44(6 * 10-5)$ & $1.58(0.00098)$ & $0.61(0.001)$ \\
\hline & $\mathrm{Cu}$ & $0.83(0.0003)$ & $0.81(3.13 * 10-5)$ & $0.63(0.0001)$ & $1.21(0.0006)$ & $0.82(0.0004)$ \\
\hline & $\mathrm{Fe}$ & $280.63(0.20)$ & $396.07(0.124)$ & $150.50(0.10)$ & $128.10(0.03)$ & $231.07(0.17)$ \\
\hline & $\mathrm{K}$ & $91.59(0.07)$ & $26.03(0.007)$ & $10.29(0.0015)$ & $89.92(0.0790$ & $53.29(0.06)$ \\
\hline & $\mathrm{Mg}$ & $336.27(0.05)$ & $0.36(0.023)$ & $324.22(0.30)$ & $309.42(0)$ & $331.21(0.044)$ \\
\hline & Mn & $5.71(0)$ & $7.52(0.0024)$ & $3.15(0.0019)$ & $5.13(0.0011)$ & $5.06(0.002)$ \\
\hline & $\mathrm{Na}$ & $1825.53(0.73)$ & $1862.39(0.52)$ & $1509.41(0.08)$ & $1349.21(0.09)$ & $1646.92(0.51)$ \\
\hline & $\mathrm{Ni}$ & $3.26(0.003)$ & $2.37(3.05 * 10-4)$ & $2.55(1.03)$ & $3.65(0.0003)$ & $2.94(0.002)$ \\
\hline & $\mathrm{Pb}$ & $8.74(0.005)$ & $7.57(0.0026)$ & $5(0.00)$ & $6.63(0.002)$ & $6.95(0.003)$ \\
\hline & $\mathrm{Zn}$ & $40.94(0.06)^{1}$ & $46.89(0.042)$ & $0.88(0.0012)$ & $6.20(0.003)$ & $21.14(0.04)$ \\
\hline & Discharge $\left(L . s^{-1}\right)$ & $135.44(26.44)^{2}$ & $106.01(22.78)$ & 104.17 (12.79) & $91.06(2.88)$ & $112.71(25.65)^{2}$ \\
\hline & TOC $\left(\mathrm{mg} . \mathrm{L}^{-1}\right)$ & $1.33(0.59)$ & $1.22(0.03)$ & $0.78(0.04)$ & $0.94(0.13)$ & $1.01(0.46)^{2}$ \\
\hline \multirow{17}{*}{ Dam } & $\mathrm{Al}$ & $211.08(0.17)$ & $52.91(0.009)$ & $37.58(0.01)$ & $33.26(0.03)$ & $97.25(0.13)$ \\
\hline & $\mathrm{Ba}$ & $45.52(0.08)$ & $69.44(0.068)$ & $3.14(0.003)$ & $3.32(0.003)$ & $28.35(0.06)$ \\
\hline & $\mathrm{Ca}$ & $1042.83(0.49)$ & $847.32(0.53)$ & $478.46(0.32)$ & $1131.86(0.04)$ & $836.96(0.48)$ \\
\hline & $\mathrm{Cd}$ & $0.30(0)$ & $0.3(0)$ & $0.42(22 * 10-5)$ & $0.3(5 * 10-5)$ & $0.35(1.3 * 10-4)$ \\
\hline & Co & $0.68(0.0002)$ & $0.50(0)$ & $0.60(0.0003)$ & $1.97(0.0015)$ & $0.84(0.00081)$ \\
\hline & $\mathrm{Cr}$ & $0.40(0.0002)$ & $0.40(0)$ & $0.40(0)$ & $1.24(0.00084)$ & $0.58(0.0005)$ \\
\hline & $\mathrm{Cu}$ & $16.07(0.03)$ & $0.60(0)$ & $0.60(0)$ & $0.99(0.0004)$ & $5.82(0.017)$ \\
\hline & $\mathrm{Fe}$ & $395.62(0.19)$ & $227.69(0.124)$ & $172.44(0.10)$ & $408.18(0.05)$ & $295.33(0.17)$ \\
\hline & $\mathrm{K}$ & $88.34(0.07)$ & $25.55(0.006)$ & $10.11(0.0022)$ & $78.45(0.071)$ & $50.16(0.06)$ \\
\hline & $\mathrm{Mg}$ & $357.53(0.1)$ & $0.35(0.034)$ & $355.35(0.35)$ & $445.39(0.01)$ & $370.78(0.077)$ \\
\hline & Mn & $8.02(0)$ & $8.25(0.0035)$ & $6.08(0.0040)$ & $13.86(0.0024)$ & $8.39(0.005)$ \\
\hline & $\mathrm{Na}$ & $2513.97(1.07)$ & $1779.24(0.50)$ & $1519.47(0.12)$ & $1458.84(0.22)$ & $1884.16(0.80)$ \\
\hline & $\mathrm{Ni}$ & $1.69(0.001)$ & $2.5(7.84 * 10-4)$ & $3.78(1.05)$ & $2.37(0.0009)$ & $2.63(0.003)$ \\
\hline & $\mathrm{Pb}$ & $6.92(0.003)$ & $8.66(0.0007)$ & $4.51(0.001)$ & $5(0.00)$ & $6.09(0.003)$ \\
\hline & $\mathrm{Zn}$ & $47.91(0.07)^{1}$ & $51.41(0.051)$ & $3.75(0.0061)$ & $4.22(0.004)$ & $24.54(0.05)$ \\
\hline & Discharge $\left(L . s^{-1}\right)$ & $90.03(37.04)^{2}$ & $53.55(1.73)$ & $29.63(6.61)$ & $22.47(0.91)$ & $52.56(35.59)^{2}$ \\
\hline & TOC $\left(\mathrm{mg} . \mathrm{L}^{-1}\right)$ & $2.17(0.81)$ & $1.47(0.12)$ & $1.28(0.19)$ & $1.64(0.24)$ & $1.71(0.65)^{2}$ \\
\hline \multirow[t]{17}{*}{ Lower Reach } & $\mathrm{Al}$ & $130.20(0.07)$ & $58.34(0.023)$ & $28.47(0.01)$ & $36.31(0.04)$ & $68.66(0.06)$ \\
\hline & $\mathrm{Ba}$ & $43.71(0.07)$ & $75.86(0.075)$ & $1.18(0.001)$ & $2.29(0.002)$ & $27.99(0.06)$ \\
\hline & $\mathrm{Ca}$ & $1040.20(0.29)$ & $1211.90(0.25)$ & $762.01(0.22)$ & $675.45(0.12)$ & $915.30(0.31)$ \\
\hline & $\mathrm{Cd}$ & $0.36(11 * 10-5)$ & $0.3(0)$ & $0.32(3 * 10-5)$ & $0.36(6 * 10-5)$ & $0.34(7 * 10-5)$ \\
\hline & Co & $1.17(0.0008)$ & $0.50(0)$ & $0.54(0.0003)$ & $2.35(0.0018)$ & $1.05(0.0011)$ \\
\hline & $\mathrm{Cr}$ & $0.40(2.01 * 10-5)$ & $0.61(2.1 * 10-4)$ & $0.33(0.00012)$ & $1.30(0.00090)$ & $0.57(0.001)$ \\
\hline & $\mathrm{Cu}$ & $101.53(0.17)$ & $0.60(0)$ & $0.58(0.0004)$ & $1.72(0.0011)$ & $34.43(0.1115)$ \\
\hline & $\mathrm{Fe}$ & $483.30(0.08)$ & $366.33(0.036)$ & $326.57(0.17)$ & $351.74(0.08)$ & $389.63(0.13)$ \\
\hline & $\mathrm{K}$ & $118.63(0.09)$ & $24.55(0.012)$ & $6.17(0.0033)$ & $108.52(0.097)$ & $63.78(0.08)$ \\
\hline & $\mathrm{Mg}$ & $406.23(0.03)$ & $0.38(0.008)$ & $355.42(0.20)$ & $381.60(0.02)$ & $380.92(0.060)$ \\
\hline & Mn & $8.91(0)$ & $10.67(0.00002)$ & $9.03(0.0026)$ & $8.09(0.0025)$ & $9.11(0.002)$ \\
\hline & $\mathrm{Na}$ & $1935.04(0.76)$ & $1922.21(0.59)$ & $1302.88(0.35)$ & $1435.33(0.22)$ & $1638.90(0.62)$ \\
\hline & $\mathrm{Ni}$ & $2.28(0.001)$ & $1.50(0)$ & $2.11(0.74)$ & $1.62(0.0001)$ & $1.98(0.001)$ \\
\hline & $\mathrm{Pb}$ & $7.65(0.004)$ & $5(0.00)$ & $5.20(0.00)$ & $6.55(0.002)$ & $6.21(0.003)$ \\
\hline & $\mathrm{Zn}$ & $38.03(0.05)^{1}$ & $50.91(0.051)$ & $0.20(0.00)$ & $6(0.006)$ & $20.79(0.04)$ \\
\hline & Discharge $\left({\left.\mathrm{L} . \mathrm{s}^{-1}\right)}\right.$ & $270.36(158.69)^{2}$ & $179.58(3.66)$ & $129.85(18.85)$ & $83.63(5.10)$ & $177.27(116.70)$ \\
\hline & TOC $\left(\mathrm{mg} . \mathrm{L}^{-1}\right)$ & $2.80(0.81)$ & $1.47(0.25)$ & $1.04(0.12)$ & $1.51(0.16)$ & $1.86(1.34)^{2}$ \\
\hline
\end{tabular}

Zn was not analyzed at May; ${ }^{2}$ Mean from March 2014 to March 2015. 


\subsection{Statistical analysis}

To assess spatial (e.g., different reaches/sites) and temporal (e.g., different seasons) changes in metal loads, DOC, $\mathrm{pH}$, and electrical conductivity over the study period, several statistical analyses were performed. Data were first tested for normal distribution using the Shapiro-Wilk test and for equal variance. Mathematical transformations such as $(\log +1)$ and $(1+x)$, were used on the stream water data for all statistical tests.

Descriptive statistics were used to establish relationships among the variables for metal loads and discharge, monthly precipitation, $\mathrm{pH}, \mathrm{EC}$ and DOC. To explore spatial and temporal influences on these variables, both parametric and nonparametric statistical tests were used. Two-way analysis of variance (ANOVA) was used on data with normal distribution and equal variance, together with pairwise multiple comparison procedures (Turkey test) to detect seasonal and spatial differences in each of the measured variables separately. Two-way ANOVA on ranks using the Kruskal-Wallis test was used to verify the significance of average differences in seasonal periods when nonparametric data were identified. Spearman correlation tests were used to verify the correlation between variables. All data were analyzed using the Minitab program (Version 17) for descriptive statistics, while SigmaPlot (Version 12) was used for ANOVA tests.

\section{RESULTS AND DISCUSSION}

\subsection{Seasonal and spatial variation of chemistry and trace metal in the streams}

Responses for most stream-water variables in both watersheds exhibited strong seasonal patterns, irrespective of reach (Figures 2, 3 and Table 3). Significant differences in mean values $(\mathrm{p} \leq 0.05)$ were observed for both reaches and seasons for the following variables: Q, Temp, $\mathrm{EC}, \mathrm{Fe}$ and $\mathrm{Mn}$ (Table 3); for DOC, $\mathrm{Ca}$ and $\mathrm{Mg}$; however, differences between means were observed only between reaches in ICU. For ISJ, only EC was found to have significant differences between reaches and seasons, while DOC, Fe, Ca, Mn show differences only between reaches. Interactions between reaches and seasons, shown in Table 3, were seen only for $\mathrm{Ca}$ and $\mathrm{Fe}$ in ICU, though these were not significant. This variation is likely due to landcover conditions and their hydrological pathways (Wickel et al., 2004), as soils of these watersheds were found to be highly permeable oxisols.

Concentrations of $\mathrm{K}$ and $\mathrm{Mn}$ were highly-to-moderately positively correlated with precipitation $(\mathrm{r}=0.732, \mathrm{p}<0.05 ; \mathrm{r}=0.478, \mathrm{p}<0.05)$ and discharge $(\mathrm{r}=0.421, \mathrm{p}<0.05 ; \mathrm{r}=0.474$, $\mathrm{p}<0.05)$ in ICU. Wickel et al. (2004), calculated the $\mathrm{Q}($ discharge $) / \mathrm{P}($ precipitation) ratio to estimated annual ET (evapotranspiration) at the watershed level and found that for all studied watersheds, the ratio for $\mathrm{K}$ had lower values, suggesting its accumulation in the system. Cu was negatively correlated with discharge $(r=-0,539, p<0.05)$, mainly at the headwater point, but positively correlated with temperature and conductivity. Fe and $\mathrm{Mn}$ showed significant responses during the rainy season, even with high discharge values (Table 3). These metals, according to Shafer et al. (1997), have different distribution constants between DOC and suspended particulate matter (SPM), giving the possibility of a transition of metals between filtrate water and particulate matter depending on conductivity and $\mathrm{pH}$.

$\mathrm{Cu}$ concentrations were high in the headwaters of ICU during the dry season and ISJ during the rainy season, indicating potential anthropogenic contamination from organic chemicals used by farmers, such as $\mathrm{CuSO} 4$, that were leached into sandy soils as a result of steep topography (e.g., Wikel et al., 2004). Another possibility is the chemical composition of rainfall. However, some authors, Shafer et al. (1997) and Bolan et al. (2014), have shown that $\mathrm{Cu}$ has a low affinity for mineral surfaces despite being strongly absorbed by organic compounds. In ICU, we observed high concentrations of $\mathrm{Cu}\left(>0.005 \mathrm{mg} . \mathrm{L}^{-1}\right)$ in the headwaters, mainly in August, with high conductivity and low DOC concentrations (Figure 3), without any correlation between copper and DOC (Table 3). In ISJ, the concentration of Cu was $0.102 \mathrm{mg} . \mathrm{L}^{-1}$ in the headwaters, 
suggesting that during the rainy season (with high discharge) these DOC-metal complexes were flushed from the soil or litter into the stream by base-flow pathways (also reported by Shafer et al., 1997).
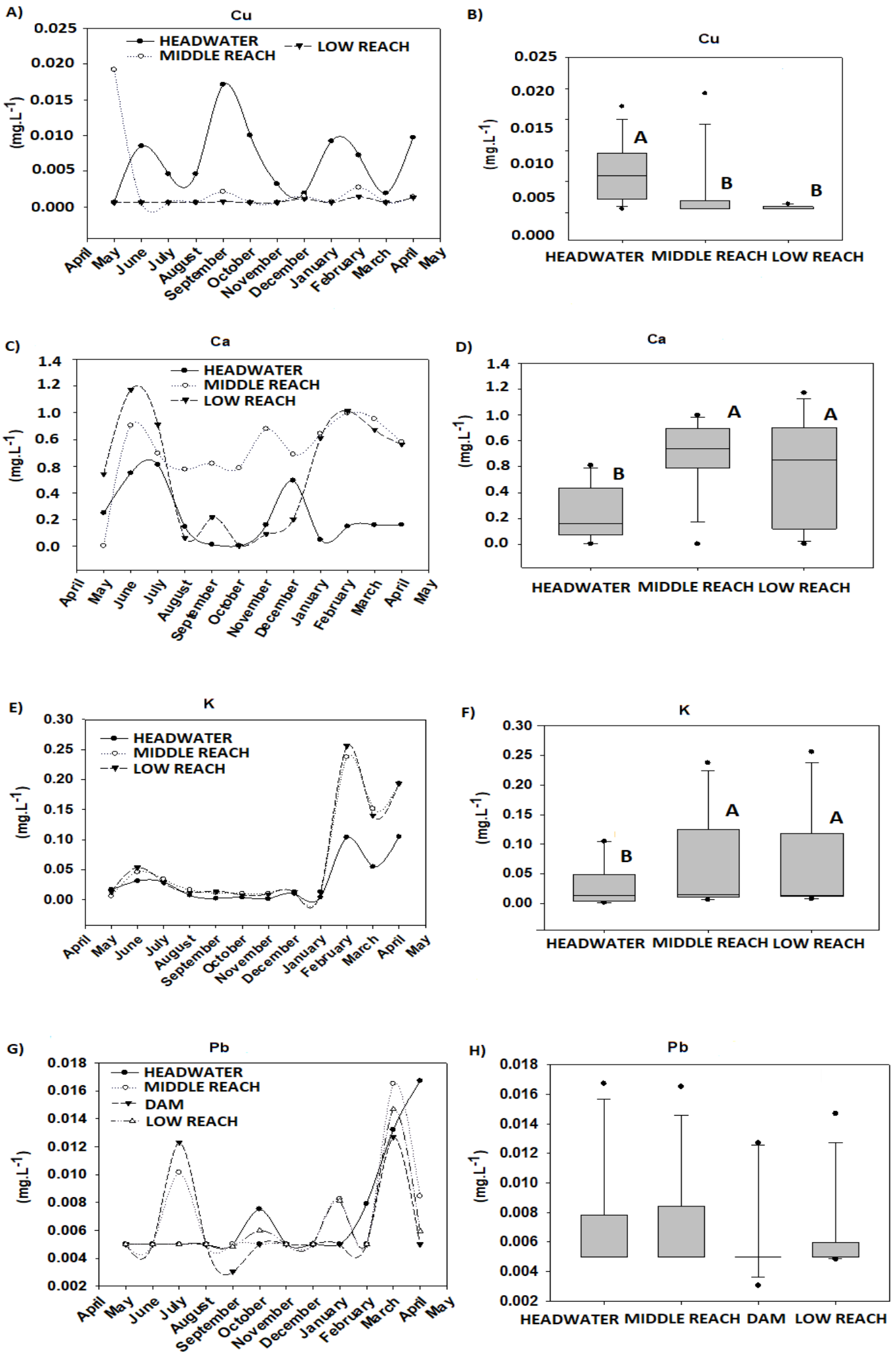

Figure 2. Spatial (a) and temporal (b) variation of copper in ICU, and dissolved metals in stream water (Ca (c) and (d) and K (e) and (f) in ICU and $\mathrm{Mn}(\mathrm{g})$, (h) and $\mathrm{Pb}(\mathrm{i})$, (j) in ISJ). Left: monitoring data; right: boxplot summarizing all data. Different letters in the boxplots indicate significant $(\mathrm{p}<0.05)$ differences among monitoring points. 

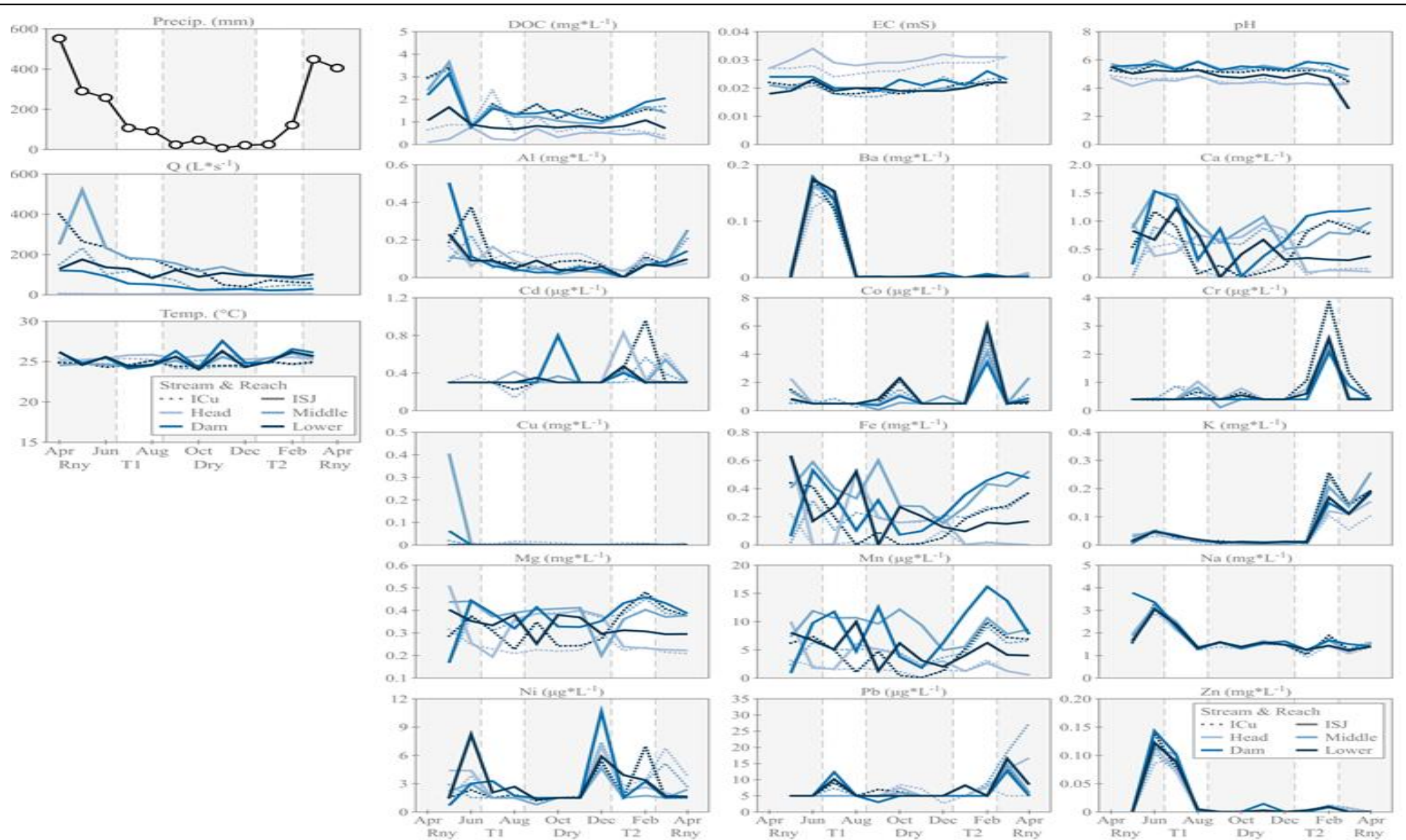

Figure 3. Temporal and spatial variation of chemical parameters. 
Table 3. Spearman coefficients for relationships between two stream chemical parameters. Strong relationships $(>0.4)$ are bold $(p<0.05)$.

\begin{tabular}{lcccccc}
\hline Metal & $\mathrm{T}^{\circ} \mathrm{C}$ & $\mathrm{pH}$ & $\mathrm{CE}$ & $\mathrm{DOC}$ & Precipitation $(\mathrm{mm})$ & $\mathrm{Q}$ \\
\hline \multicolumn{7}{c}{} \\
\hline $\mathrm{Al}$ & 0.264 & -0.111 & 0.275 & -0.215 & $\mathbf{0 . 4 4 7}$ & -0.04 \\
$\mathrm{Ca}$ & -0.384 & $\mathbf{0 . 4 7 7}$ & -0.169 & 0.336 & 0.253 & 0.314 \\
$\mathrm{Cu}$ & $\mathbf{0 . 5 1 6}$ & -0.398 & $\mathbf{0 . 4 2 0}$ & -0.264 & -0.074 & $\mathbf{- 0 . 5 3 9}$ \\
$\mathrm{Fe}$ & $\mathbf{- 0 . 4 0 5}$ & $\mathbf{0 . 4 0 8}$ & -0.191 & 0.324 & 0.358 & $\mathbf{0 . 4 4}$ \\
$\mathrm{K}$ & -0.036 & 0.14 & 0.031 & 0.218 & $\mathbf{0 . 7 2 2}$ & 0.229 \\
$\mathrm{Mg}$ & $\mathbf{- 0 . 5 5 2}$ & 0.414 & -0.306 & 0.353 & 0.045 & 0.347 \\
$\mathrm{Mn}$ & -0.387 & 0.388 & -0.239 & 0.433 & $\mathbf{0 . 4 3 8}$ & $\mathbf{0 . 4 9 7}$ \\
\hline & & \multicolumn{5}{c}{ ISJ } \\
\hline $\mathrm{Al}$ & 0.111 & -0.169 & 0.294 & -0.013 & $\mathbf{0 . 6 7 6}$ & 0.092 \\
$\mathrm{Ca}$ & -0.046 & 0.366 & -0.139 & 0.23 & 0.093 & 0.212 \\
$\mathrm{Cu}$ & 0.02 & -0.237 & 0.389 & 0.219 & 0.22 & -0.199 \\
$\mathrm{Fe}$ & -0.09 & 0.349 & -0.29 & 0.302 & 0.198 & 0.322 \\
$\mathrm{~K}$ & 0.129 & -0.158 & 0.353 & 0.04 & $\mathbf{0 . 7 9 8}$ & -0.073 \\
$\mathrm{Mg}$ & 0.016 & 0.351 & -0.182 & 0.294 & -0.004 & 0.183 \\
$\mathrm{Mn}$ & -0.108 & $\mathbf{0 . 4 8 4}$ & -0.344 & $\mathbf{0 . 4 6 6}$ & 0.142 & 0.329 \\
\hline
\end{tabular}

Further, DOC mobility may be driven by $\mathrm{pH}$ and $\mathrm{EC}$, as DOC is influenced by ionic strength and was found to be negatively correlated with EC. In the headwater reaches, ionic strength was high and DOC concentration was low, likely due to lower $\mathrm{pH}$ (4.15-4.22) than at the middle reach (5.56-5.28), lower reach (5.66-5.96) and dam (5.5) (Table 2). We also observed weak correlations between metals and DOC $(\mathrm{r}<0.2)$. A possible explanation is that metals are linked in the particulate phase rather than in the aqueous phase in these streams, except for $\mathrm{Mn}$, which showed a moderate response to DOC $(r=0.466, p=0.001)$ and was the only metal to behave this way, mainly at the dam point in January, February and March.

Significant positive correlations between precipitation and $\mathrm{Al}(\mathrm{r}=0.676, \mathrm{p}<0.05)$ and precipitation and $\mathrm{K}(\mathrm{r}=0.798, \mathrm{p}<0.05)$ were observed in the middle reach, lower reach, and dam at ISJ (Table 3). In this small watershed, pasture and bare soil have more influence on metal behavior than in ICU, as metal concentrations increased with discharge after rainfall events probably due to runoff events. ICU also had a higher percentage of forested and secondary forested areas, in which metal and DOC fluxes were found to have lower responses than in ISJ under storm-flow conditions (Table 1).

As expected, discharge was highest during the rainy season in all reaches of both streams, with a gradual decline throughout the sampling period until the start of the next rainy season (Figure 4).

DOC followed a similar pattern as discharge in both streams, with considerable variability in DOC concentrations found during the rainy season relative to other seasons, which suggests that periodic rain episodes deliver DOC to streams. While DOC was highly variable across each of the study reaches, concentrations also were generally higher in the lower reaches of ICU and ISJ (Table 2).

Temperature, EC and $\mathrm{pH}$ fluctuated throughout the sampling period, with EC increasing slightly over the course of the sampling period from the rainy season to the dry season and back again. In contrast, $\mathrm{pH}$ remained consistent across the sampling period in all reaches until the start of the next rainy season (in March 2015), when it dropped abruptly in all reaches of both streams. Higher variation in $\mathrm{pH}$ appeared in the headwaters of both streams, given that these areas contained more abundant riparian vegetation and secondary vegetation in the surrounding areas (Table 2). 


\section{(a)}

\section{Lower Reach Cumaru}
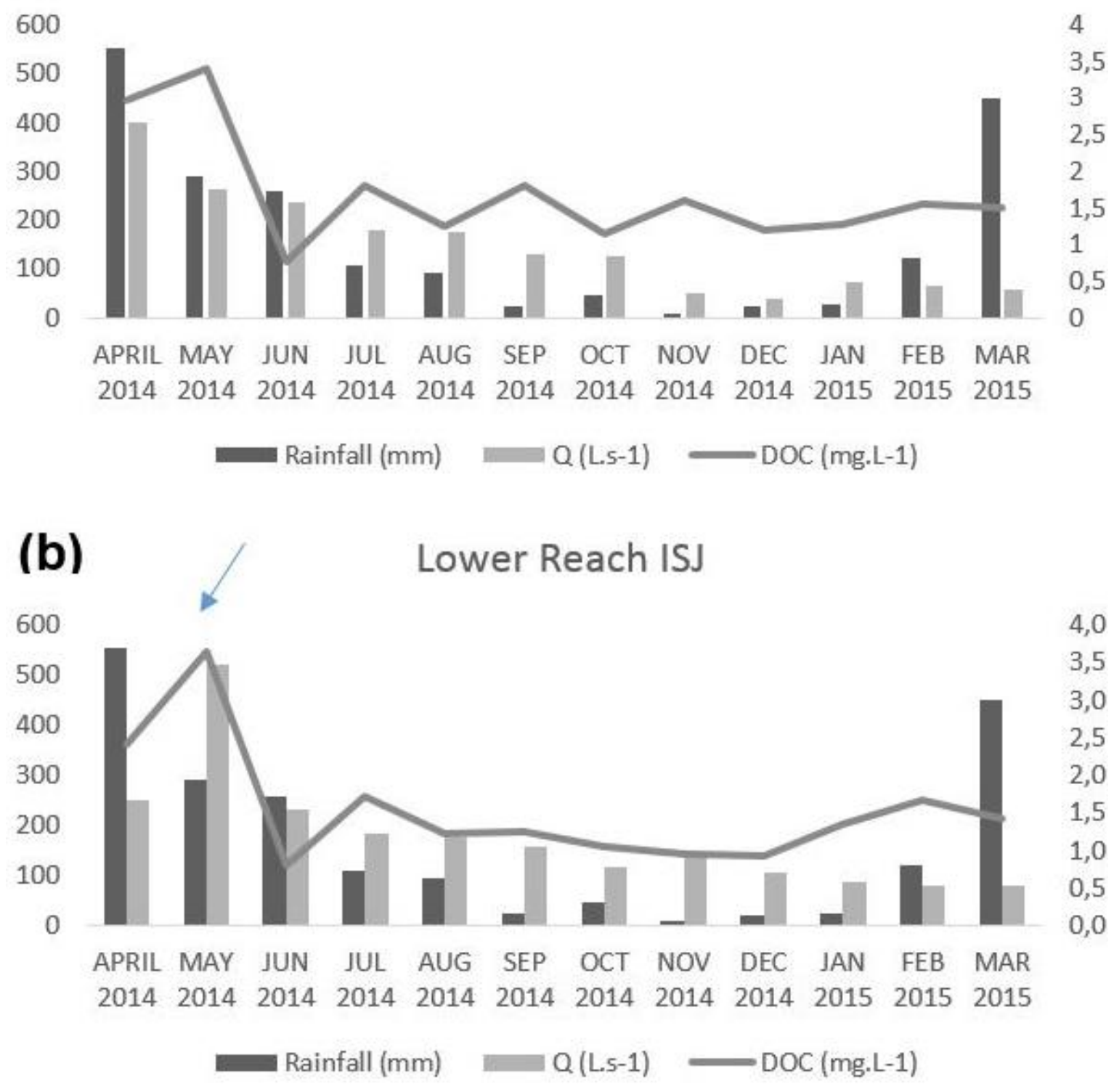

Figure 4. Discharge $(\mathrm{Q})$, rainfall $(\mathrm{mm})$ and dissolved organic carbon (DOC) temporal variability, (a) Cumaru Stream and (b) São João Stream.

A seasonal influence across stream reaches was found for several variables (Figure 2). In general, these patterns were marked by precipitous changes occurring in the transitions to and from the rainy season: a decrease in concentrations from the rainy season to T1 for $\mathrm{Al}, \mathrm{Na}, \mathrm{Ba}$, $\mathrm{Zn}$, and $\mathrm{Cu}$ and an increase in concentrations from $\mathrm{T} 2$ to the next rainy season for $\mathrm{Co}, \mathrm{Cr}, \mathrm{K}$, $\mathrm{Ni}$, and $\mathrm{Pb}$ (Figure 3 and Table 2). For $\mathrm{Cu}$, however, this result may have been driven by a single, high outlier. There also was some intra-seasonal variability for several trace metals, particularly for $\mathrm{Cd}, \mathrm{Co}, \mathrm{Cr}$, and $\mathrm{Ni}$, though this result was not consistent across reaches.

The remaining elements and variables $(\mathrm{Ca}, \mathrm{Fe}, \mathrm{Mg}$, and $\mathrm{Mn}$ ) showed complex spatial and temporal relationships across the study period. The highest concentrations of $\mathrm{Ca}, \mathrm{Fe}$, and $\mathrm{Mn}$ generally were found in the rainy and transition (T1 and T2) seasons, and typically increased from the headwater reaches downstream to the dam and/or middle- and lower reaches (Figure 2). However, while $\mathrm{Mg}$ demonstrated the same seasonal patterns as $\mathrm{Ca}, \mathrm{Fe}$, and $\mathrm{Mn}$, its spatial pattern was evident only in ICU (Figure 2 and Table 4). 
Table 4. Significance (p-value) of two-factor ANOVAs of the two study watersheds, Cumaru (ICU) and São João (ISJ), showing results for reach (headwater, middle, and lower for ICU and headwater, middle, dam, and lower for ISJ), season (rainy, T1, dry, T2), and their interactions. Temp=temperature.

\begin{tabular}{ccccccc}
\hline \multirow{2}{*}{ Parameter } & \multicolumn{3}{c}{$\mathrm{ICu}$} & & & ISJ \\
\cline { 2 - 7 } & Reach & Season & Inter. & Reach & Season & Inter. \\
\hline $\mathrm{Q}$ & $<\mathbf{0 . 0 0 1}$ & $\mathbf{0 . 0 2 0}$ & 0.959 & $*$ & $*$ & $*$ \\
$\mathrm{Temp}$. & $<\mathbf{0 . 0 0 1}$ & $<\mathbf{0 . 0 0 1}$ & 0.595 & 0.346 & 0.171 & 0.601 \\
$\mathrm{pH}$ & $*$ & $*$ & $*$ & $*$ & $*$ & $*$ \\
$\mathrm{EC}$ & $<\mathbf{0 . 0 0 1}$ & $<\mathbf{0 . 0 0 1}$ & 0.887 & $<\mathbf{0 . 0 0 1}$ & $\mathbf{0 . 0 0 7}$ & 0.529 \\
$\mathrm{DOC}$ & $\mathbf{0 . 0 0 6}$ & 0.517 & 0.786 & $<\mathbf{0 . 0 0 1}$ & $*$ & $*$ \\
$\mathrm{Al}$ & 0.446 & $\mathbf{0 . 0 1 0}$ & 0.337 & 0.376 & $*$ & $*$ \\
$\mathrm{Ba}$ & $*$ & $*$ & $*$ & $*$ & $*$ & $*$ \\
$\mathrm{Ca}$ & $<\mathbf{0 . 0 0 1}$ & 0.085 & 0.068 & $\mathbf{0 . 0 1 0}$ & 0.151 & 0.071 \\
$\mathrm{Cd}$ & $*$ & $*$ & $*$ & $*$ & $*$ & $*$ \\
$\mathrm{Co}$ & $*$ & $*$ & $*$ & $*$ & $*$ & $*$ \\
$\mathrm{Cr}$ & $*$ & $*$ & $*$ & $*$ & $*$ & $*$ \\
$\mathrm{Cu}$ & $*$ & $*$ & $*$ & $*$ & $*$ & $*$ \\
$\mathrm{Fe}$ & $<\mathbf{0 . 0 0 1}$ & $\mathbf{0 . 0 0 4}$ & $\mathbf{0 . 0 3 3}$ & $\mathbf{0 . 0 4 0}$ & 0.216 & 0.668 \\
$\mathrm{~K}$ & 0.557 & 0.031 & $*$ & 0.994 & $*$ & $*$ \\
$\mathrm{Mg}$ & $<\mathbf{0 . 0 0 1}$ & $*$ & $*$ & 0.040 & $*$ & $*$ \\
$\mathrm{Mn}$ & $<\mathbf{0 . 0 0 1}$ & $\mathbf{0 . 0 0 1}$ & 0.121 & $<\mathbf{0 . 0 0 1}$ & 0.440 & 0.529 \\
$\mathrm{Na}$ & 0.816 & 0.250 & 1.000 & $*$ & $*$ & $*$ \\
$\mathrm{Ni}$ & $*$ & $*$ & $*$ & $*$ & $*$ & $*$ \\
$\mathrm{~Pb}$ & $*$ & $*$ & $*$ & $*$ & $*$ & $*$ \\
$\mathrm{Zn}$ & $*$ & $*$ & $*$ & $*$ & $*$ & $*$ \\
\hline
\end{tabular}

* Nonparametric data.

Seasonal and spatial variations of $\mathrm{Ca}$ and $\mathrm{K}$ were significant in the lower- and middle reaches, with different mean concentrations compared to the headwaters, likely because of changes in $\mathrm{pH}$. Lower $\mathrm{Ca}$ and $\mathrm{K}$ values were measured during smaller discharge and precipitation periods in the transition from rainy to dry season and the dry season. This differs from the results of Barroso (2011), who also studied small Amazonian watersheds and found high concentrations during the dry season. However, this pattern is similar to results found by Markewitz et al. (2011) for other small Amazonian watersheds, likely due to the influence of land cover on discharge-concentration relationships (e.g., runoff) (Markewitz et al., 2011).

Ca showed strong positive correlations with $\mathrm{Fe}(\mathrm{r}=0.795, \mathrm{p}<0.05), \mathrm{K}(\mathrm{r}=0.66, \mathrm{p}<0.05), \mathrm{Mg}$ $(\mathrm{r}=0.845, \mathrm{p}<0.05)$, and $\mathrm{Mn}(\mathrm{r}=0.803, \mathrm{p}<0.05)$, and negative correlation with $\mathrm{Cu}(\mathrm{r}=-0.52$, $\mathrm{p}<0.05$ ), which seems to come from the same source, soil origin. It is probable that $\mathrm{Ca}$ forms a metal solution complex mainly with fine sediments and DOC, as Ca had the opposite behavior to $\mathrm{Cu}$, which had lower concentrations with increased DOC concentrations and decreased specific conductivity (Table 5 and 6). The highest concentrations of $\mathrm{Ca}$ in transition seasons can be related to burning of fallow vegetation to prepare land for crops and/or pastures in the rainy season; as noted by Wickel et al. (2004), this land management can promote calcium input to streams by overland flow.

Concentrations of $\mathrm{K}$ showed a relevant concentration in the middle- and lower-stream reaches of both watersheds during the wet season, probably because of the influence of nearby burned areas and pasture. On the other hand, in ISJ, $\mathrm{Pb}$ and $\mathrm{Mn}$ were found to have higher concentrations at the dam during the dry season and in the transition from dry to rainy season, probably due to a strong influence from anthropogenic activities (bathing, washing clothes). Mn was influenced by both $\mathrm{pH}$ and DOC $(\mathrm{r}=0.484, \mathrm{p}=0.001$ and $\mathrm{r}=0.466, \mathrm{p}=0.001$, respectively), while $\mathrm{Pb}$ concentrations above the legal limit $\left(0.01 \mathrm{mg} . \mathrm{L}^{-1}\right.$ established by CONAMA Resolution 357, for stream water Class 1) (CONAMA, 2005) were found in all sampled reaches of ISJ, mainly during the wet season due to household activities. Figure 3 
illustrates the upstream-downstream trends and temporal variations of $\mathrm{Ca}$ and $\mathrm{K}$ ions.

Overall, metal concentrations ( $\mu \mathrm{g} . \mathrm{L}^{-1}$ ) exhibited strong seasonal patterns, in the order of $\mathrm{Na}>\mathrm{Ca}>\mathrm{Fe}>\mathrm{Mg}>\mathrm{Al}>\mathrm{Zn}>\mathrm{K}$, though $\mathrm{Fe}$ concentrations can be at least in part attributed to geogenic and pedogenic origins (Figure 5). $\mathrm{Cu}, \mathrm{Cd}, \mathrm{Co}, \mathrm{Ni}$ and $\mathrm{Pb}$ all were found in relatively low concentrations $\left(<0.006 \mathrm{mg} . \mathrm{L}^{-1}\right)$. In both watersheds, higher concentrations of $\mathrm{Al}$ were measured in May, when the $\mathrm{pH}$ was low and precipitation and discharge were high. Al was moderately positively correlated with $\mathrm{Ba}, \mathrm{Cu}, \mathrm{Na}$ and $\mathrm{K}$ in ISJ, while DOC was positively correlated with $\mathrm{Fe}, \mathrm{Mg}$, and $\mathrm{Mn}$. This suggests partitioning of changes from solid phase to aqueous phase, accounting for the response with fulvic and humic compounds that can complex with metals. In both streams, DOC also was positively correlated $\mathrm{Ca}, \mathrm{Fe}, \mathrm{Mg}, \mathrm{Mn}$ and $\mathrm{Na}$ $(p<0.05)$. DOC and organic substances (agrochemicals) may interfere in the fluxes of these metals by binding with them, thereby decreasing the concentrations in a soluble phase (Cakmak et al., 2009). In these watersheds the agrochemical "Roundup" (glyphosate) is frequently used by the small farms.

We also saw similar behavior both spatially and temporary, particularly in the middle and lower reaches in the rainy-dry transition and dry seasons, with a strong influence of discharge and $\mathrm{pH}$. During the dry-rainy transition season, the response in the lower reach was similar to that in the headwaters, likely due to decreased discharge during this time. Temperature, conductivity, and $\mathrm{Cu}$ concentrations also were more variable in the headwater reaches, mainly during the dry season. Some authors have indicated that $\mathrm{Cu}, \mathrm{Zn}$, and $\mathrm{Cd}$ are associated with farmlands that traditionally use organic fertilizers, such as animal manure and sewage sludge (Gupta and Charles, 1999; Antonious et al., 2008). However, in the ICU headwaters, stream water can receive leached metals from riparian vegetation found on nearby slopes, where soils can be acidified by rainwater, thus contributing to metal loads by increasing the rates of bedrock dissolution (e.g., Yager et al., 2013). Overall, the influence of discharge on the metal loads was clear: decreased discharge during the dry season resulted in similar variation in water chemistry in the headwaters of ICU as in the lower reaches (Figure 6).

Additionally, precipitation contributed to high metal loads, as $\mathrm{K}$ and $\mathrm{Mn}$ were positively correlated with precipitation, and Fe was strongly correlated with discharge and moderately correlated with $\mathrm{pH}$ in ICU. Yu et al. (2014), observed significant temporal variability in metal loads in stream water and noted that rainfall appears to be the key driver of variability across several scales. In ISJ, the middle reach, lower reach, and dam showed larger variability in conductivity, mainly during the dry season. On the other hand, Fe and $\mathrm{Mn}$ concentrations increased during the rainy season due to precipitation events that increased discharge. The stream at the headwater point of ISJ receives significant quantity of leaves from riparian vegetation in steep terrain, blocking channel flow and resulting in additional inputs of some metals, such as K, Fe and Mn. It seems that in the headwaters of ISJ, some metal elements, such as $\mathrm{Pb}, \mathrm{Al}, \mathrm{Co}$ and $\mathrm{Cr}$, enter the stream during transition seasons. This phenomenon can likely be explained by anthropogenic effects (washing dishes and clothes, as well as agricultural inputs).

We also noted that land cover has a strong influence on the chemical composition of baseflow stream water, but it is not the principal factor affecting metal loads. Instead, the strong factor is the intense precipitation events in areas with high soil erosion, as in the lower reaches. The vegetation around the headwaters retains metals and DOC, preventing their export by river discharge compared to downstream reaches, where the main land cover is a mixture of agriculture, bare soil, and pasture. Dissolved metals in both watersheds, however, were dependent on $\mathrm{pH}, \mathrm{DOC}, \mathrm{Al}$, and $\mathrm{Fe}$, together with variability in rainfall intensity and differences in land cover. The total dissolved metals in rainwater samples could be an important source of these metals, mainly $\mathrm{Cu}, \mathrm{Al}, \mathrm{K}$ and $\mathrm{Pb}$ in the watersheds, and should be monitored in future studies. 
Table 5. Spearman Correlation for Cumaru Watershed Data.

\begin{tabular}{|c|c|c|c|c|c|c|c|c|c|c|c|c|c|c|c|c|c|c|c|c|}
\hline & $\mathbf{T}$ & pH & CE & DOC & Al & $\mathbf{B a}$ & $\mathrm{Ca}$ & Cd & Co & $\mathrm{Cr}$ & $\mathrm{Cu}$ & $\mathrm{Fe}$ & $\mathbf{K}$ & Mg & Mn & $\mathrm{Na}$ & $\mathrm{Ni}$ & $\mathbf{P b}$ & $\mathbf{Z n}$ & PPT \\
\hline \multirow[t]{2}{*}{ pH } & -0.616 & & & & & & & & & & & & & & & & & & & \\
\hline & 0.000 & & & & & & & & & & & & & & & & & & & \\
\hline \multirow[t]{2}{*}{ CE } & 0.703 & -0.679 & & & & & & & & & & & & & & & & & & \\
\hline & 0.000 & 0.000 & & & & & & & & & & & & & & & & & & \\
\hline \multirow[t]{2}{*}{ DOC } & -0.468 & 0.456 & -0.601 & & & & & & & & & & & & & & & & & \\
\hline & 0.004 & 0.005 & 0.000 & & & & & & & & & & & & & & & & & \\
\hline \multirow[t]{2}{*}{ Al } & 0.264 & -0.111 & 0.275 & -0.215 & & & & & & & & & & & & & & & & \\
\hline & 0.138 & 0.539 & 0.121 & 0.229 & & & & & & & & & & & & & & & & \\
\hline \multirow[t]{2}{*}{ Ba } & -0.157 & 0.285 & -0.043 & 0.112 & 0.274 & & & & & & & & & & & & & & & \\
\hline & 0.382 & 0.108 & 0.814 & 0.536 & 0.106 & & & & & & & & & & & & & & & \\
\hline \multirow[t]{2}{*}{$\mathrm{Ca}$} & -0.384 & 0.477 & -0.169 & $\mathbf{0 . 3 3 6}$ & -0.009 & 0.493 & & & & & & & & & & & & & & \\
\hline & 0.027 & 0.005 & 0.347 & 0.056 & 0.96 & 0.002 & & & & & & & & & & & & & & \\
\hline \multirow[t]{2}{*}{ Cd } & 0.033 & -0.136 & 0.221 & 0.081 & -0.116 & 0.097 & 0.353 & & & & & & & & & & & & & \\
\hline & 0.854 & 0.452 & 0.216 & 0.656 & 0.502 & 0.572 & 0.035 & & & & & & & & & & & & & \\
\hline \multirow[t]{2}{*}{ Co } & -0.133 & -0.091 & 0.005 & 0.089 & 0.179 & -0.063 & -0.123 & 0.32 & & & & & & & & & & & & \\
\hline & 0.462 & 0.614 & 0.978 & 0.624 & 0.295 & 0.716 & 0.476 & 0.057 & & & & & & & & & & & & \\
\hline \multirow[t]{2}{*}{$\mathrm{Cr}$} & 0.188 & 0.041 & -0.099 & 0.167 & -0.192 & -0.091 & 0.131 & 0.166 & 0.248 & & & & & & & & & & & \\
\hline & 0.295 & 0.821 & 0.584 & 0.354 & 0.261 & 0.596 & 0.445 & 0.334 & 0.145 & & & & & & & & & & & \\
\hline \multirow[t]{2}{*}{$\mathrm{Cu}$} & 0.516 & -0.398 & 0.420 & -0.264 & 0.119 & -0.051 & -0.520 & 0.03 & 0.22 & -0.016 & & & & & & & & & & \\
\hline & 0.002 & 0.022 & 0.015 & 0.138 & 0.491 & 0.77 & 0.001 & 0.861 & 0.198 & 0.928 & & & & & & & & & & \\
\hline \multirow[t]{2}{*}{$\mathrm{Fe}$} & -0.405 & 0.408 & -0.191 & 0.324 & 0.236 & 0.314 & 0.795 & 0.181 & -0.124 & -0.018 & -0.526 & & & & & & & & & \\
\hline & 0.02 & 0.019 & 0.286 & 0.066 & 0.166 & 0.062 & 0.000 & 0.291 & 0.471 & 0.918 & 0.001 & & & & & & & & & \\
\hline \multirow[t]{2}{*}{$\mathbf{K}$} & -0.036 & 0.140 & 0.031 & 0.218 & 0.264 & 0.423 & 0.660 & 0.467 & 0.121 & 0.226 & -0.256 & 0.574 & & & & & & & & \\
\hline & 0.844 & 0.438 & 0.863 & 0.224 & 0.120 & 0.010 & 0.000 & 0.004 & 0.481 & 0.185 & 0.131 & 0.000 & & & & & & & & \\
\hline \multirow{2}{*}{ Mg } & -0.552 & 0.414 & -0.306 & $\mathbf{0 . 3 5 3}$ & -0.214 & 0.222 & 0.845 & 0.378 & -0.016 & 0.253 & -0.613 & 0.757 & 0.478 & & & & & & & \\
\hline & 0.001 & 0.017 & 0.083 & 0.044 & 0.211 & 0.194 & 0.000 & 0.023 & 0.926 & 0.137 & 0.000 & 0.000 & 0.003 & & & & & & & \\
\hline \multirow[t]{2}{*}{ Mn } & -0.387 & 0.388 & -0.239 & 0.433 & 0.131 & 0.333 & 0.803 & 0.339 & 0.093 & 0.191 & -0.387 & 0.888 & 0.659 & 0.782 & & & & & & \\
\hline & 0.026 & 0.026 & 0.18 & 0.012 & 0.447 & 0.047 & 0.000 & 0.043 & 0.590 & 0.265 & 0.020 & 0.000 & 0.000 & 0.000 & & & & & & \\
\hline \multirow[t]{2}{*}{$\mathrm{Na}$} & -0.312 & 0.407 & -0.22 & 0.332 & 0.399 & 0.825 & 0.401 & 0.062 & 0.147 & -0.213 & -0.135 & 0.348 & 0.334 & 0.182 & 0.348 & & & & & \\
\hline & 0.077 & 0.019 & 0.218 & 0.059 & 0.016 & 0.000 & 0.015 & 0.721 & 0.391 & 0.212 & 0.433 & 0.037 & 0.046 & 0.289 & 0.038 & & & & & \\
\hline \multirow[t]{2}{*}{$\mathbf{N i}$} & 0.132 & -0.109 & 0.378 & -0.315 & -0.046 & 0.162 & 0.13 & 0.431 & 0.103 & -0.088 & 0.223 & 0.000 & 0.296 & 0.004 & -0.003 & 0.015 & & & & \\
\hline & 0.465 & 0.546 & 0.03 & 0.074 & 0.79 & 0.344 & 0.45 & 0.009 & 0.55 & 0.611 & 0.191 & 1.000 & 0.08 & 0.981 & 0.985 & 0.932 & & & & \\
\hline \multirow[t]{2}{*}{$\mathbf{P b}$} & -0.044 & -0.072 & -0.038 & 0.214 & 0.091 & 0.029 & 0.166 & 0.148 & 0.297 & 0.192 & -0.134 & 0.141 & 0.300 & 0.148 & 0.229 & -0.006 & -0.190 & & & \\
\hline & 0.807 & 0.692 & 0.832 & 0.232 & 0.6 & 0.865 & 0.333 & 0.391 & 0.079 & 0.261 & 0.437 & 0.414 & 0.076 & 0.39 & 0.179 & 0.973 & 0.267 & & & \\
\hline \multirow[t]{2}{*}{ Zn } & 0.291 & 0.084 & 0.202 & -0.177 & 0.236 & 0.496 & 0.339 & 0.244 & -0.047 & 0.197 & 0.106 & 0.015 & 0.402 & -0.009 & 0.173 & 0.304 & 0.244 & 0.139 & & \\
\hline & 0.1 & 0.641 & 0.26 & 0.324 & 0.165 & 0.002 & 0.043 & 0.152 & 0.786 & 0.25 & 0.539 & 0.932 & 0.015 & 0.96 & 0.313 & 0.072 & 0.151 & 0.42 & & \\
\hline PPT & 0.261 & 0 & 0.183 & 0.18 & 0.447 & 0.101 & 0.253 & 0.231 & 0.185 & 0.05 & -0.074 & 0.358 & 0.722 & 0.045 & 0.438 & 0.16 & 0.175 & 0.264 & 0.224 & \\
\hline & 0.124 & 0.998 & 0.285 & 0.294 & 0.006 & 0.558 & 0.137 & 0.176 & 0.28 & 0.773 & 0.669 & 0.032 & 0.000 & 0.795 & 0.007 & 0.35 & 0.307 & 0.119 & 0.189 & \\
\hline $\mathbf{Q}$ & -0.588 & 0.661 & -0.776 & 0.668 & -0.04 & 0.137 & 0.314 & -0.124 & -0.001 & -0.013 & -0.539 & 0.44 & 0.229 & 0.347 & 0.497 & 0.374 & -0.207 & 0.098 & -0.096 & 0.225 \\
\hline & 0.000 & 0.000 & 0.000 & 0.000 & 0.825 & 0.448 & 0.076 & 0.491 & 0.997 & 0.941 & 0.001 & 0.01 & 0.200 & 0.048 & 0.003 & 0.032 & 0.248 & 0.587 & 0.594 & 0.194 \\
\hline
\end{tabular}


Table 6. Spearman Correlation for São João Watershed Data.

\begin{tabular}{|c|c|c|c|c|c|c|c|c|c|c|c|c|c|c|c|c|c|c|c|c|}
\hline & $\mathbf{T}\left(\mathbf{C}^{\circ}\right)$ & pH & EC & DOC & Al & $\mathbf{B a}$ & $\mathrm{Ca}$ & Cd & Co & $\mathrm{Cr}$ & $\mathrm{Cu}$ & $\mathbf{F e}$ & $\mathbf{K}$ & Mg & Mn & $\mathbf{N a}$ & $\mathbf{N i}$ & $\mathbf{P b}$ & $\mathrm{Zn}$ & PPT \\
\hline \multirow[t]{2}{*}{ pH } & -0.205 & & & & & & & & & & & & & & & & & & & \\
\hline & 0.161 & & & & & & & & & & & & & & & & & & & \\
\hline \multirow[t]{2}{*}{ EC } & 0.288 & -0.415 & & & & & & & & & & & & & & & & & & \\
\hline & 0.047 & 0.003 & & & & & & & & & & & & & & & & & & \\
\hline \multirow{2}{*}{ DOC } & -0.082 & 0.685 & -0.407 & & & & & & & & & & & & & & & & & \\
\hline & 0.579 & 0.000 & 0.004 & & & & & & & & & & & & & & & & & \\
\hline \multirow{2}{*}{ Al } & 0.111 & -0.169 & 0.294 & -0.013 & & & & & & & & & & & & & & & & \\
\hline & 0.474 & 0.272 & 0.052 & 0.933 & & & & & & & & & & & & & & & & \\
\hline \multirow[t]{2}{*}{$\mathbf{B a}$} & 0.132 & 0.016 & 0.182 & -0.054 & 0.502 & & & & & & & & & & & & & & & \\
\hline & 0.391 & 0.917 & 0.237 & 0.728 & 0.000 & & & & & & & & & & & & & & & \\
\hline \multirow[t]{2}{*}{$\mathrm{Ca}$} & -0.046 & 0.366 & -0.139 & 0.230 & 0.170 & 0.401 & & & & & & & & & & & & & & \\
\hline & 0.767 & 0.015 & 0.367 & 0.133 & 0.247 & 0.005 & & & & & & & & & & & & & & \\
\hline \multirow[t]{2}{*}{ Cd } & -0.050 & -0.001 & 0.073 & -0.015 & -0.300 & -0.546 & -0.238 & & & & & & & & & & & & & \\
\hline & 0.745 & 0.995 & 0.637 & 0.921 & 0.038 & 0.000 & 0.103 & & & & & & & & & & & & & \\
\hline \multirow[t]{2}{*}{ Co } & -0.035 & -0.158 & 0.178 & 0.068 & 0.345 & -0.032 & -0.169 & -0.113 & & & & & & & & & & & & \\
\hline & 0.822 & 0.306 & 0.249 & 0.66 & 0.016 & 0.829 & 0.252 & 0.446 & & & & & & & & & & & & \\
\hline \multirow[t]{2}{*}{$\mathrm{Cr}$} & 0.258 & -0.113 & 0.247 & -0.065 & 0.037 & -0.094 & 0.029 & 0.199 & 0.359 & & & & & & & & & & & \\
\hline & 0.091 & 0.466 & 0.106 & 0.673 & 0.802 & 0.525 & 0.843 & 0.175 & 0.012 & & & & & & & & & & & \\
\hline \multirow[t]{2}{*}{$\mathrm{Cu}$} & 0.020 & -0.237 & 0.389 & -0.219 & 0.454 & 0.338 & -0.094 & -0.313 & 0.484 & 0.122 & & & & & & & & & & \\
\hline & 0.897 & 0.122 & 0.009 & 0.153 & 0.001 & 0.019 & 0.523 & 0.030 & 0.000 & 0.408 & & & & & & & & & & \\
\hline \multirow[t]{2}{*}{$\mathrm{Fe}$} & -0.090 & 0.349 & -0.290 & 0.302 & 0.221 & 0.225 & 0.801 & -0.163 & -0.056 & 0.115 & -0.113 & & & & & & & & & \\
\hline & 0.560 & 0.020 & 0.056 & 0.047 & 0.131 & 0.124 & 0.000 & 0.269 & 0.704 & 0.436 & 0.443 & & & & & & & & & \\
\hline \multirow[t]{2}{*}{$\mathbf{K}$} & 0.129 & -0.158 & 0.353 & 0.040 & 0.648 & 0.371 & 0.111 & -0.205 & 0.321 & 0.306 & 0.303 & 0.196 & & & & & & & & \\
\hline & 0.404 & 0.307 & 0.019 & 0.799 & 0.000 & 0.009 & 0.452 & 0.161 & 0.026 & 0.034 & 0.036 & 0.181 & & & & & & & & \\
\hline \multirow[t]{2}{*}{ Mg } & 0.016 & 0.351 & -0.182 & 0.294 & -0.015 & 0.203 & 0.810 & -0.144 & -0.075 & 0.070 & -0.199 & 0.833 & -0.02 & & & & & & & \\
\hline & 0.918 & 0.019 & 0.238 & 0.053 & 0.917 & 0.166 & 0.000 & 0.327 & 0.614 & 0.638 & 0.176 & 0.000 & 0.894 & & & & & & & \\
\hline \multirow{2}{*}{ Mn } & -0.108 & 0.484 & -0.344 & 0.466 & -0.021 & 0.170 & 0.772 & -0.096 & -0.07 & 0.202 & -0.228 & 0.844 & 0.134 & 0.827 & & & & & & \\
\hline & 0.486 & 0.001 & 0.022 & 0.001 & 0.886 & 0.248 & 0.000 & 0.515 & 0.638 & 0.169 & 0.119 & 0.000 & 0.362 & 0.000 & & & & & & \\
\hline \multirow[t]{2}{*}{$\mathbf{N a}$} & 0.049 & 0.170 & 0.076 & 0.165 & 0.542 & 0.825 & 0.379 & -0.529 & -0.024 & -0.259 & 0.306 & 0.193 & 0.125 & 0.216 & 0.129 & & & & & \\
\hline & 0.753 & 0.271 & 0.622 & 0.283 & 0.000 & 0.000 & 0.008 & 0.000 & 0.870 & 0.076 & 0.034 & 0.189 & 0.398 & 0.141 & 0.384 & & & & & \\
\hline \multirow[t]{2}{*}{$\mathbf{N i}$} & -0.130 & -0.141 & 0.239 & -0.224 & 0.104 & 0.371 & 0.081 & -0.208 & 0.126 & 0.161 & 0.364 & -0.001 & 0.365 & -0.052 & 0.058 & 0.133 & & & & \\
\hline & 0.400 & 0.36 & 0.118 & 0.145 & 0.482 & 0.009 & 0.582 & 0.155 & 0.392 & 0.276 & 0.011 & 0.995 & 0.011 & 0.725 & 0.694 & 0.369 & & & & \\
\hline \multirow[t]{2}{*}{$\mathbf{P b}$} & -0.053 & -0.244 & 0.111 & -0.099 & 0.085 & -0.254 & -0.106 & 0.267 & 0.152 & 0.183 & -0.078 & -0.192 & 0.381 & -0.267 & -0.151 & -0.346 & 0.058 & & & \\
\hline & 0.732 & 0.111 & 0.474 & 0.522 & 0.566 & 0.082 & 0.473 & 0.067 & 0.303 & 0.213 & 0.599 & 0.19 & 0.008 & 0.067 & 0.307 & 0.016 & 0.696 & & & \\
\hline \multirow[t]{2}{*}{ Zn } & 0.193 & 0.068 & 0.197 & -0.131 & 0.142 & 0.434 & 0.137 & -0.030 & -0.162 & 0.231 & 0.059 & -0.043 & 0.413 & -0.142 & 0.038 & 0.205 & 0.326 & 0.155 & & \\
\hline & 0.209 & 0.661 & 0.201 & 0.396 & 0.334 & 0.002 & 0.353 & 0.838 & 0.273 & 0.114 & 0.690 & 0.77 & 0.004 & 0.336 & 0.795 & 0.162 & 0.024 & 0.292 & & \\
\hline PPT & 0.005 & 0.048 & 0.223 & 0.194 & 0.676 & 0.123 & 0.093 & -0.092 & 0.363 & 0.201 & 0.220 & 0.198 & 0.798 & -0.004 & 0.142 & 0.082 & 0.121 & 0.475 & 0.154 & \\
\hline & 0.974 & 0.747 & 0.127 & 0.187 & 0.000 & 0.403 & 0.530 & 0.534 & 0.011 & 0.171 & 0.132 & 0.177 & 0.000 & 0.979 & 0.334 & 0.578 & 0.411 & 0.001 & 0.294 & \\
\hline $\mathbf{Q}$ & -0.298 & 0.537 & -0.702 & 0.526 & 0.092 & 0.013 & 0.212 & -0.174 & -0.044 & -0.188 & -0.199 & 0.322 & -0.073 & 0.183 & 0.329 & 0.197 & -0.120 & -0.068 & -0.110 & 0.135 \\
\hline & 0.040 & 0.000 & 0.000 & 0.000 & 0.552 & 0.936 & 0.166 & 0.259 & 0.777 & 0.222 & 0.194 & 0.033 & 0.639 & 0.235 & 0.029 & 0.199 & 0.438 & 0.660 & 0.476 & 0.362 \\
\hline
\end{tabular}


(a)
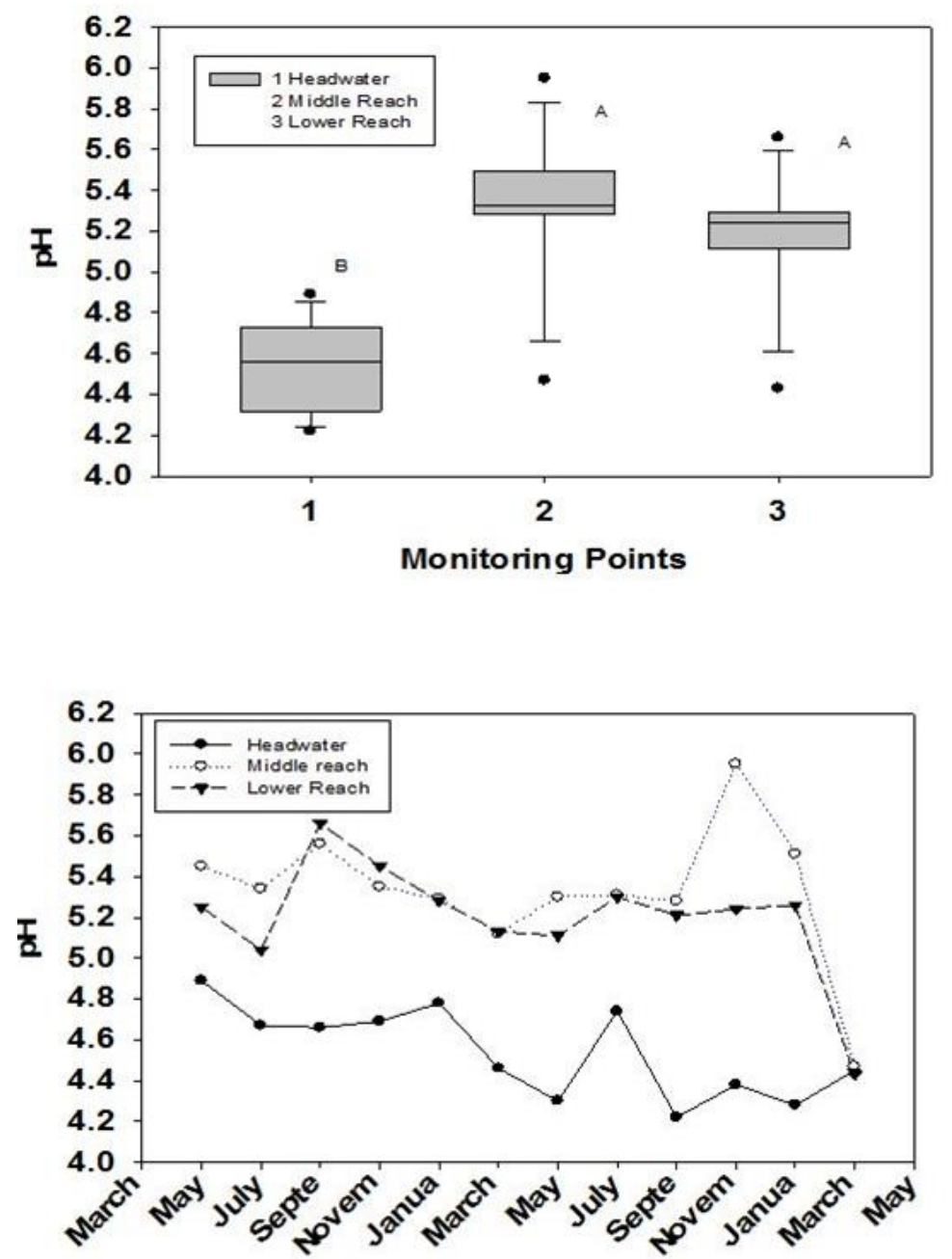

April 2014 to March 2015 (b)

IgSJ
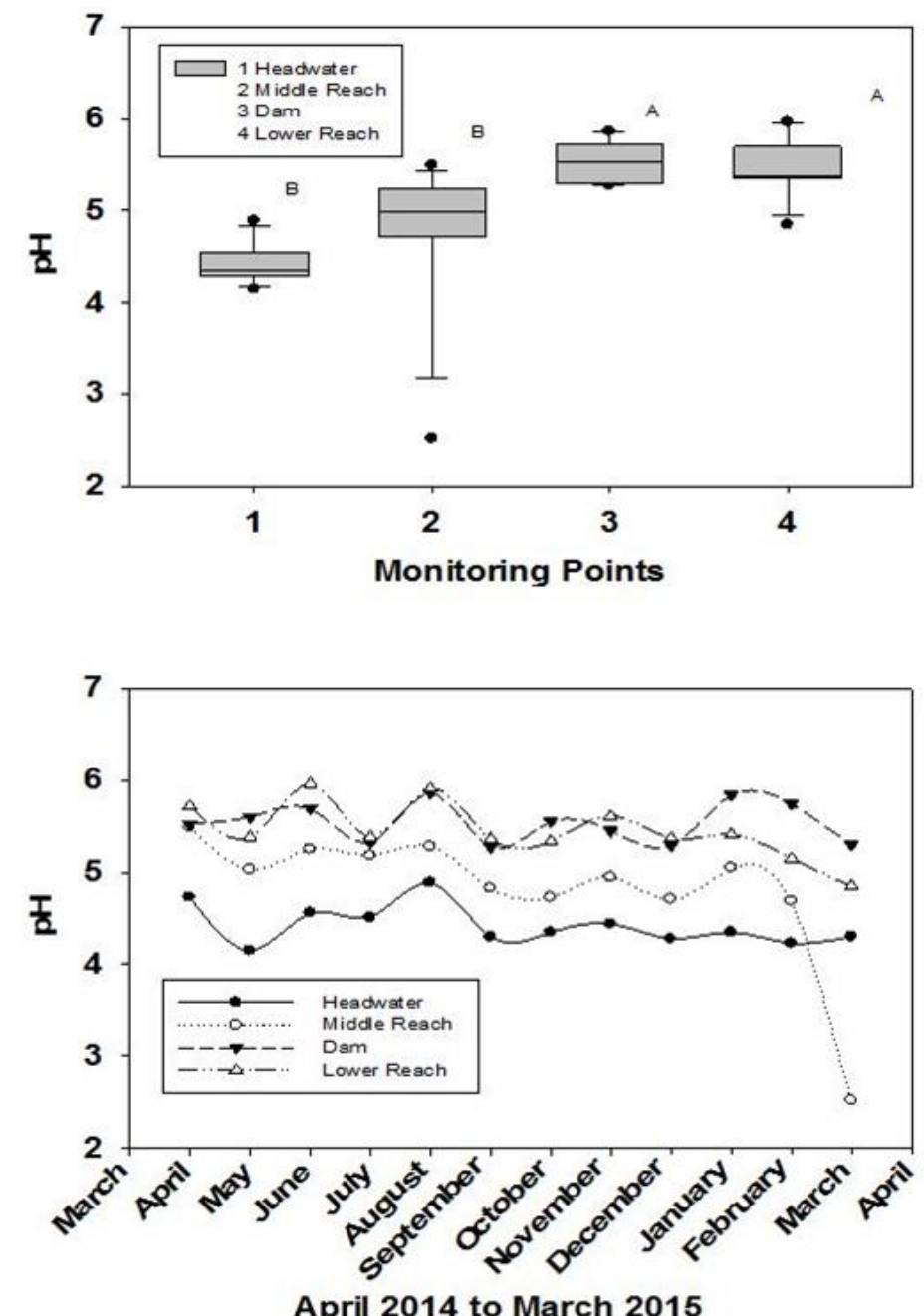

Figure 5. The $\mathrm{pH}$ in stream water at three sampling points in ICU and at four sampling points in ISJ. Different letters in the boxplots indicate significant $(\mathrm{p}<0.05)$ differences between the monitoring points. 
(a)

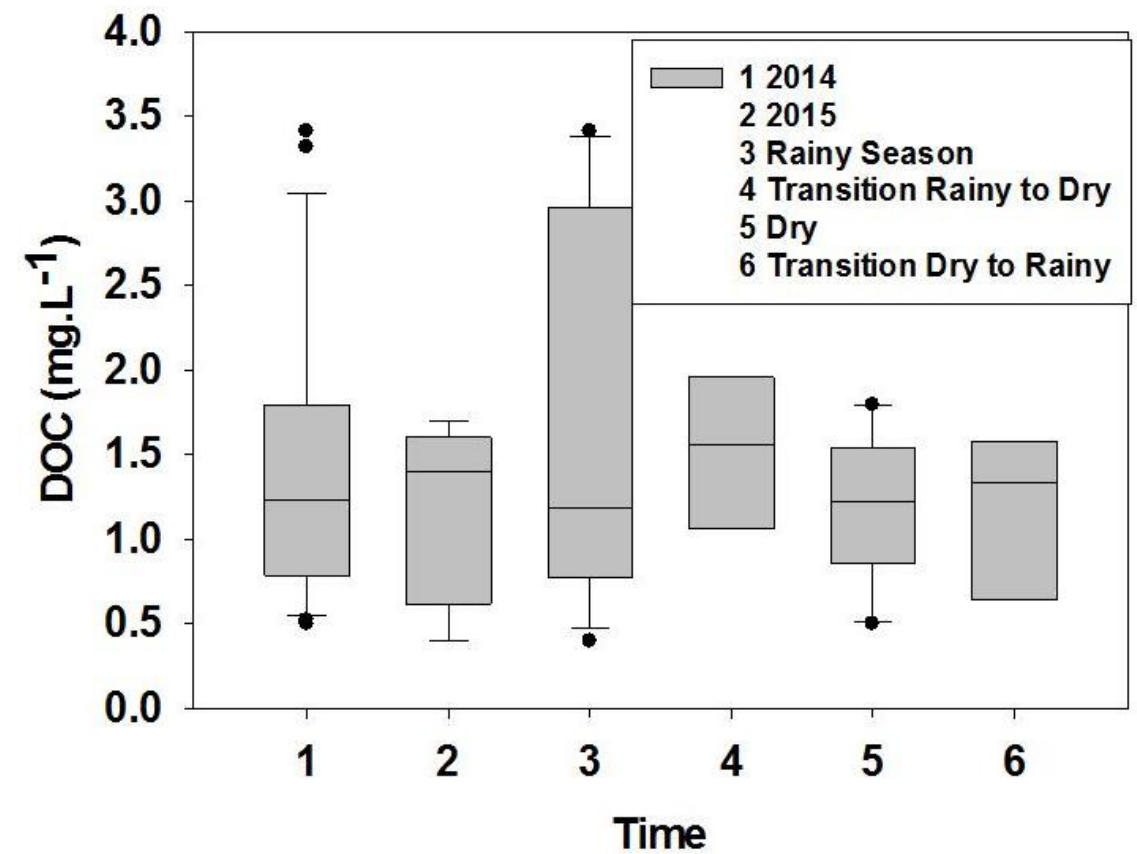

(b)

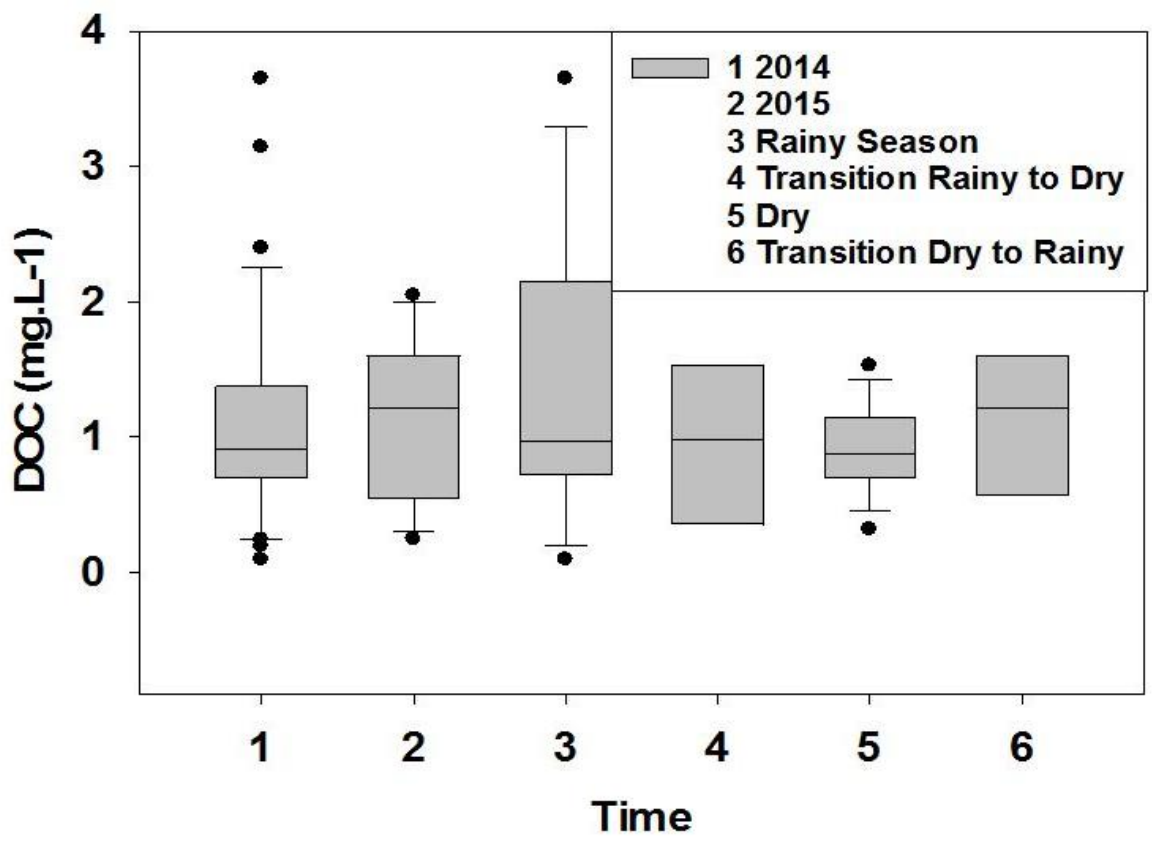

Figure 6. DOC temporal variation of small watersheds at ICU (a) and ISJ (b). 


\subsection{Factors controlling the fluxes of water chemistry and dissolved metals in streams}

When considering metal fluxes across the different stream reaches and watersheds, we found that ISJ consistently exported more metals than ICU, likely because of increased pasture and agriculture in the watershed and a decreased buffer effect from riparian vegetation, releasing more nutrients to the streams. The lower reaches had the highest flux values, due to other downstream water channel inputs, as predicted (Table 7). These areas varied considerably with local factors such as flowpath, soil properties, topography and site-to-stream distance.

For the dissolved form of carbon (DOC), flux measurements (Equation 1) in downstream reaches were considerably greater than in headwaters as result of inputs from nearby agriculture and pasture. As shown in Table 1, ICU was comprised of $49.08 \%$ secondary forest, $24.61 \%$ pasture, and $6.02 \%$ annual agriculture, while ISJ had $36.12 \%, 34.65 \%$ and $3.08 \%$, respectively. Therefore, the total export flux (Table 7) was larger in these more-impacted areas in ISJ, with significant fluxes (in Kg.month ${ }^{-1} \cdot$ ha $^{-1}$ ) of metals (Ca, Na, Mg, Fe, Mn) in all seasons, especially during the rainy season. While pasture led to increased nutrient- and metal export, the presence of natural secondary vegetation reduced metal and DOC export, especially in the headwater reaches. Other studies in eastern Amazonia have observed greater leaching by water departments (river, runoff, underground water) of nutrients (mainly $\mathrm{Ca}$ ) in watersheds occupied by slash-and-burn agriculture than those where chop-and-mulching was adopted (Wickel, 2004; Barroso, 2011). In ICU and ISJ, smallholders previously burned fallow vegetation to cultivate perennial crops, enriching the sandy soils with cations that are rapidly leached until reaching stream water. While perennial agriculture comprised $4-7 \%$ of land cover in both ICU and ISJ (Table 1), larger export occurred in ISJ due to its increased area in pasture. This result is similar to Figueiredo et al. $(2010 ; 2013)$, who observed increased sodium with increasing crop cover area in nearby streams. Lower metal and DOC fluxes in headwaters can be related to an increased amount of secondary forest or forest nearby, but lower fluxes were also observed at the dam. This was likely a result of high sedimentation, since metals and DOC tend to be absorbed by suspended particulate material. The importance of secondary forest was also found by Wickel et al. (2004), as it helped mitigate the transfer of nutrients and pollutants to streams due to the protective action of the root network.

Table 7. Chemical fluxes of selected variables from each reach of the two watersheds, Cumaru (ICU) and São João (ISJ).

\begin{tabular}{|c|c|c|c|c|}
\hline $\begin{array}{c}\text { Fm ICU } \\
\left(\text { Kg.month }^{-1} \cdot \text { ha }^{-1}\right)\end{array}$ & Headwater & Middle Reach & Low Reach & No Dam \\
\hline DOC & 0.0000015 & 0.0001 & 0.0002 & \\
\hline $\mathrm{Ca}$ & 0.41 & 36.52 & 52.26 & \\
\hline $\mathrm{Na}$ & 2.80 & 85.81 & 156.87 & \\
\hline $\mathrm{Mg}$ & 0.43 & 18.63 & 31.13 & \\
\hline $\mathrm{Fe}$ & 0.06 & 10.70 & 18.04 & \\
\hline $\mathrm{Mn}$ & 0.00 & 0.26 & 0.43 & \\
\hline $\mathrm{K}$ & 0.06 & 3.18 & 5.92 & \\
\hline $\mathrm{Al}$ & 0.19 & 4.42 & 11.33 & \\
\hline $\begin{array}{c}\text { Fm ISJ } \\
\left(\text { Kg.month }^{-1} \cdot \text { ha }^{-1}\right)\end{array}$ & Headwater & Middle Reach & Low Reach & Dam \\
\hline DOC & $1.3 \mathrm{E}-06$ & 0.0001 & 0.0003 & $8.8 \mathrm{E}-05$ \\
\hline $\mathrm{Ca}$ & 1.74 & $\mathbf{5 7 . 5 0}$ & 159.02 & 43.11 \\
\hline $\mathrm{Na}$ & 5.65 & 181.92 & 284.73 & 97.05 \\
\hline $\mathrm{Mg}$ & 1.06 & 36.59 & 66.18 & 19.10 \\
\hline $\mathrm{Fe}$ & 0.53 & 25.52 & 67.69 & 15.21 \\
\hline $\mathrm{Mn}$ & $1.1 \mathrm{E}-02$ & 0.56 & 1.58 & 0.43 \\
\hline $\mathrm{K}$ & 0.16 & 5.89 & 11.08 & 2.58 \\
\hline $\mathrm{Al}$ & 0.28 & 8.47 & 11.93 & 5.01 \\
\hline
\end{tabular}




\section{CONCLUSIONS}

This analysis demonstrated key spatial and temporal patterns in metals and DOC, all of which were considerably influenced by both seasonal differences and anthropogenic activity. The presence of forest or secondary vegetation in the headwater areas was a key factor mitigating the responses we observed, indicating that these types of land cover help to increase resilience to changes in discharge or extreme hydrological events. One of the key outcomes of this work is the need for increased sediment and particulate matter monitoring in small streams such as these to characterize sensitive changes in water quality. This should be conducted together with chemical analysis of rainwater to detect inorganic and organic chemicals from different sources. The availability of an automatic system to collect water samples from these streams, especially during storm events, also would greatly benefit the understanding of biogeochemical changes in a tropical area used for more than one hundred years. As we hypothesized, land cover plays an important role in DOC, dissolved metals, and their interactions, as a result of the anthropogenic impacts related to agriculture activities. Furthermore, our results demonstrate the need for increased education efforts and local policies to mitigate pollution from the improper- or untreated disposal of human sewage, as well as to help promote a transition in land use from slash-and-burn agriculture to more sustainable practices (e.g., chopping and dispersion of biomass in the soil). Such measures can help to preserve forest and the secondary vegetation, as well as minimize nutrient loss from soil and sediments, thereby avoiding release of heavy metals and organic substances into small streams.

\section{ACKNOWLEDGMENTS}

We gratefully acknowledge the funding of the Agrohidro Project of Embrapa (Brazilian Agricultural Research Corporation - 01120100103).

\section{REFERENCES}

ANTONIOUS, F. G.; TURLEY, E. T.; SIKORA, F.; SNYDER, J. C. Heavy metal mobility in runoff water and absorption by eggplant fruits from sludge treated soil. Journal of Environmental Science Health B, v. 43, n. 6, p. 526-532, 2008. https://doi.org/10.1080/03601230802174748

APHA; AWWA; WEF. Standard Methods for the Examination of Water and Wastewater. 20. ed. Washington, 1998. 1325p.

AITKENHEAD-PETERSON, J. A.; MCDOWELL, W. H.; NEFF, J. C. Sources, production and regulation of allochthonous dissolved organic matter. In: FINDLAY, S.; SINSABAUGH, R. L. (eds.). Aquatic ecosystems, interactivity of dissolved organic matter. Amsterdam: Academic Press, 2003. p. 26-70. https://doi.org/10.1016/B978012256371-3/50003-2

BARROSO, D. F. R. Hydrogeochemical fluxes in fluvial waters of micro-basins of northeastern Pará and its relationship with land use. 2011. 222p. Dissertation (Master's degree in Environmental Science) - Federal University of Pará, Belém, 2011.

BOLAN, N.; KUNHIKRISHAN, A.; THANGARAJAN, R.; KUMPIENE, J.; PARK, J.; TOMOYUKI, M.; KIRKHAM, M. B. Remediation of heavy metal(loid)s contaminated soils - To mobilize or to immobilize? Journal of Hazardous Materials, v. 266, p. 141166, 2014. http://dx.doi.org/10.1016/j.jhazmat.2013.12.018 
CAKMAK, I.; YAZICI, A.; TUTUS, Y.; OZTURK, L. Glyphosate reduced seed and leaf concentrations of calcium, manganese, magnesium, and iron in non-glyphosate resistant soybean. European Journal of Agronomy, v. 31, p. 114-119, 2009. https://doi.org/10.1016/j.eja.2009.07.001

CAK, A. D.; MORAN, E. F.; FIGUEIREDO, R. O.; LU, D.; LI, G.; HETRICK, S. Urbanization and small household agricultural land use choices in the Brazilian Amazon and the role for the water chemistry of small streams. Journal of Land Use Science, v. 11, n. 2, p. 203-221, 2016. https://doi.org/10.1080/1747423X.2015.1047909

CONAMA (Brasil). Resolução n 357 de 17 de março de 2005. Dispõe sobre a classificação dos corpos de água e diretrizes ambientais para o seu enquadramento, bem como estabelece as condições e padrões de lançamento de efluentes, e dá outras providências. Diário Oficial [da] União: seção 1, Brasília, DF, n. 053, p. 58-63, 18 mar. 2005.

DAS, B.; NORDIN, R.; MAZUMBER, A. Watershed land use as a determinant of metal concentrations in freshwater systems. Environ Geochem Health, v. 31, n. 6, p. 595-607, 2009. https://doi.org/10.1007/s10653-008-9244-z

DUANN, S.; HE, Y.; KAUSHAL, S. S.; BIANCHI, T. S.; WARD, N. W.; GUO, L. Impact of wetland decline on decreasing dissolved organic carbon concentrations along the Mississippi River Continuum. Frontiers in Marine Science, v. 3, n. 280, 2017. https://doi.org/10.3389/fmars.2016.00280

FIGUEIREDO, R. O; BÖRNER, J.; DAVIDSON, E. A. Watershed services payments to smallholders in the Brazilian Amazon: Challenges and perspectives. Journal Environment \& Water, v. 9, n. 2, p. 6-17, 2013. http://dx.doi.org/10.4136/ambiagua. 1056

FIGUEIREDO, R.O.; MARKEWITZ, D.; DAVIDSON, E.A.; SCHULER, A.E.; WATRIN, O.S.; SILVA, P.S. Land-use effects on the chemical attributes of low-order streams in the eastern Amazon. Journal of Geophysical Research: Biogeosciences, v. 115, n. G4, 2010. http://dx.doi.org/10.1029/2009JG001200

GLOBAL WATER INSTRUMENTATION. FP101-FP201 Global Flow Probe: User's Manual. Rancho Cordova: Global Water Instrumentation, Inc, 2004. 15 p.

GUPTA, G.; CHARLES, S. Trace elements in soils fertilized with poultry litter. Poultry Science, v. 78, n. 12, p. 1695-1698, 1999. https://doi.org/10.1093/ps/78.12.1695

HUSER, B. J.; KÖHLER, S. J.; WILANDER, A.; FÖLSTER, J. Temporal and spatial trends for trace metals in streams and rivers across Sweden (1996-2009). Biogeosciences Discussions, v. 8, p. 1813-1823, 2011. https://doi.org/10.5194/bg-8-1813-2011

JANEAU, J. L.; GILLARD, L. C.; GRELLIER, S.; JOUQUET, P.; LE, T. P. Q.; LUU, T. N. M.; NGO, Q. A.; ORANGE, D.; PHAM, D. R.; TRAN, D. T.; TRAN, S. H.; TRINH, A. D.; VALENTIN, C.; ROCHELLE-NEWALL, E. Soil erosion, dissolved organic carbon and nutrient losses under different land uses systems in a small catchment in northern Vietnam. Agricultural Water Management, v. 146, p. 314-323, 2014. https://doi.org/10.1016/j.agwat.2014.09.006

KATO, O. R.; KATO, M. S. A.; SÁ, T. D. A.; FIGUEIREDO, R. O. No-tillage in capoeira. Journal Science \& Environment, v. 29, p. 99-111, 2004. 
LIMA, L. M.; SOUZA, E. L.; FIGUEIREDO, R. O. Dimethoate retention and its relation to $\mathrm{pH}$, clay and organic matter contents by sediments in non saturated zone of a small catchment of the northeast Pará. Acta Amazonica, v. 37, n. 2, p. 187-194, 2007. http://dx.doi.org/10.1590/S0044-59672007000200003

MARKEWITZ, D.; LAMMON III, E. C.; BUSTAMANTE, M. C.; CHAVES, J.; FIGUEIREDO, R. O.; JOHNSON, M. S.; KRUSCHE, A.; NEILL, C.; SILVA, J. S. O. Discharge-calcium concentrations relationships in stream of the Amazon and Cerrado of Brazil: soil or land use controlled. Biogeochemistry, v. 105, p. 19-35, 2011. https://doi.org/10.1007/s10533-011-9574-2

SHAFER, M. M.; OVERDIER, J. T.; HURLEY, J. P.; ARMSTRONG, H. D.; WEBB, D. The influence of dissolved organic carbon, suspended particulates, and hydrology on the concentration, partitioning and variability of trace metals in two contrasting Wisconsin Watersheds (U.S.A.). Chemical Geology, v. 136, n. 27, p. 71-97, 1997. https://doi.org/10.1016/S0009-2541(96)00139-8

STUMM, W.; MORGAN, J. J. Aquatic chemistry: Chemical balances and natural water rates. 3 ed. New York: Wiley, 1996. 1022p.

WICKEL, B. A. J. Water and Nutrient Dynamics of a Humid Tropical Watershed in Eastern Amazonia. Bonn: Center of Development Research/University of Bonn, 2004. 135p.

YAGER, D. B.; JOHNSON, R. H.; ROCKWELL, B. W.; CAINE, J. S.; SMITH, K. S. A GIS and statistical approach to identify variables that control water quality in hydrothermally altered and mineralized watersheds, Silverton, Colorado, USA. Environment Earth Science, v. 70, n. 3, p. 1057-1082, 2013. https://doi.org/10.1007/s12665-013-2229-y

YU, S.; WU, Q.; LI, Q.; GAO, J.; LIN, Q.; MA, J.; XU, Q.; WU, S. Anthropogenic land uses elevated metals levels in stream water in an urbanizing watershed. Science of the Total $\begin{array}{llllll}\text { Environment, } & \text { v. } & 488-489, & \text { p. } & 61-69, & \end{array}$ https://dx.doi.org/10.1016/j.scitotenv2014.04.061 